\title{
Improving the Construction of the DBM Over Approximation of the State Space of Real-time Preemptive Systems*
}

\author{
Abdelli Abdelkrim ${ }^{\dagger}$
}

\begin{abstract}
We present in this paper an algorithm allowing an efficient computation of the tightest $D B M$ over-approximation of the state space of preemptive systems modeled by using Time Petri Nets with inhibitor arcs. First of all, we propose an algorithm that reduces the effort of computing the tightest $D B M$ over-approximated graph. For this effect, each class of this graph is expressed as a pair $(M, \widetilde{D})$, where $M$ is a marking and $\widetilde{D}$ is the system of all $D B M$ inequalities even the redundant ones. We thereby make it possible to compute the system $\widetilde{D}$ straightforwardly in its normal form, without requiring to compute the intermediary polyhedra. Hence, we succeed to remove the errors reported in the implementation of other $D B M$ approximations. Then we show that by relaxing a bit in the precision of the $D B M$ approximation, we can achieve to construct more compact graphs while reducing still more the cost of their computation. We provide for this abstraction a suitable equivalence relation that contract yet more the graphs. The experimental results comparing the defined constructions with other approaches are reported.
\end{abstract}

Keywords: Preemptive Systems, Time Petri Nets, Stopwatch Inhibitor arcs, State class graph, DBM over-approximation

\section{Introduction}

Nowadays, real-time systems are becoming more and more complex and are often critical. Therefore, their verification has to be performed thoroughly in order to prove the correctness of their behaviors. These systems consist of several tasks that are interacting and sharing one or more resources (e.g processors, memory). Hence, the problem is to determine, for instance, whether these actions can be scheduled in such a way that their constraints are satisfied.

Furthermore, the correctness proofs of such systems are demanding much theory regarding their increasing complexity. We may need, for instance, to consider formal

\footnotetext{
${ }^{*}$ This work was supported by the Algerian national Project PNR number 8/u160/3067

${ }^{\dagger}$ LSI Laboratory- Computer Science Department- USTHB University-Algiers Algeria
} 
models requiring the specification of time preemption; concept where execution of a task may be stopped for a while and later resumed at the same point. This notion of suspension requires to extend the semantics of timed clocks in order to handle such behaviors. For this effect, Cassez et al have introduced the stopwatch mechanism $[10]$ and hence many models have been defined, as for instance, hybrid automata $(L H A)[2]$ and stopwatch automata $(S W A)$ [10]. Time Petri nets $(T P N)$ have also been considered in several works including Preemptive-TPN [7], Stopwatch-TPN [5], Inhibitor-TPN [15], and Scheduling-TPN [13].

The verification of qualitative and quantitative properties of such a system on its formal description involves the investigation of a part of or the whole set of its reachable states that determines its state space. As the state space is generally infinite due to dense time semantics, we need therefore to compute finite abstractions of it, that preserve properties of interest. In these abstractions, states are grouped together, in order to obtain a finite number of these groups. These groups of states are, for instance, regions and zones for timed automata, or state classes[4] for time Petri nets. Hence, the states pertaining to each group can be described by a system of linear inequalities, noted $D$, whose set of solutions determines the state space of the group. Hence, if the model does not use any stopwatch, then $D$ is of a particular form, called DBM (Difference Bound Matrix) [8]. However, when using stopwatches, the system $D$ becomes more complex and does not fit anymore into a $D B M$. In actual fact, $D$ takes a general polyhedral form whose canonical form [1] is given as a conjunction of two subsystems $D=\widetilde{D} \wedge \widehat{D}$, where $\widetilde{D}$ is a $D B M$ system and $\widehat{D}$ is a polyhedral system that cannot be encoded with $D B M s$.

The major shortcoming of manipulating polyhedra is the performance loss in terms of computation speed and memory usage. Indeed, the complexity of solving a general polyhedral system is exponential in the worst case, while it is polynomial for a $D B M$ system. Furthermore, the reachability is proved to be undecidable for both $S W A$ and $L H A$ [10] [2][11], as well as for TPN extended with stopwatches [5] even when the net is bounded. As a consequence, the finiteness of the graph cannot be guaranteed.

In order to speed up the state space computation, an idea is to leave out the subsystem $\widehat{D}$, to keep only the system $\widetilde{D}$ approximating thus the space of $D$ to the $D B M$ containing it, see [7][15] for details. The obvious consequence of the overapproximation is that we add states in the computed group that are not reachable indeed. However, this could prevent the graph computation to terminate, by making the number of computed markings unbounded. Conversely, this can also make the computation of the approximated graph terminate by cutting off the polyhedral inequalities that prevent the convergence. It is noteworthy that since the resulted graph encompasses the exact one, only a subset of properties of interest are preserved.

In order to perform efficiently the exact analysis of the over-approximated graph, Bucci et al [7] have proposed to use the $D B M$ over-approximation as a pre-computing before cleaning up the graph from its additional sequences that have been added due to over-approximation. This is done by constraining each sequence reachable in the over-approximated graph by a linear system that reproduces the 
original timing constraints of the model. Hence, if there is no solution that makes the sequence be firable according to the time constraints of the system, then the sequence has been introduced by the over-approximation and can be cleared up, otherwise the solution set makes it possible to determine the feasible timings of this sequence.

Furthermore, in order to settle a compromise between both techniques, a hybrid approach has been proposed by Roux et al [14]. The latter puts forward a sufficient condition that determines the cases where the subsystem $\widehat{D}$ becomes redundant in $D$. Hence, the combination of both $D B M$ and polyhedral representations makes it possible to build the exact state class graph faster and with lower expenses in terms of memory usage comparatively to the polyhedra based approach [13]. More recently, Berthomieu et al have proposed an over-approximation method based on a quantization of the polyhedral system $D[5]$. The latter approach ends in the exact computation of the graph in almost all cases faster than the hybrid approach [14]. Nevertheless, this technique is more costly in terms of computation time and memory usage comparatively to $D B M$ over-approximation although it yields much precise graphs.

We consider in this paper real time preemptive systems modeled by using ITPN (Time Petri Nets with inhibitor arcs) [15]. This model extends TPN with inhibitor arcs to control the progression and the suspension of stopwatches.

First of all, we propose a new algorithm to compute the tightest $D B M$ overapproximation of the state class graph of preemptive systems. For this effect, we express each class, noted $\widetilde{E}$, of the approximated graph as a pair $(M, \widetilde{D})$ where $M$ is a marking and $\widetilde{D}$ is the system of all $D B M$ constraints, even the redundant ones. We show that by maintaining a complete representation of $\widetilde{D}$ and avoiding to compute its minimal form, we achieve to define an efficient algorithm that computes a normalized class in a square time in the number of enabled transitions. Besides, we prove that the systems $\widetilde{D}$ and $\widehat{D}$ are equivalent; this ensures that $\widetilde{D}$ is the tightest $D B M$ over-approximation that one can derive from $D$. Unlike the other approaches [15][7], our algorithm avoids the computation of the intermediary polyhedra $D$. We thereby avoid its computation and its manipulation and remove all the costs induced by the derivation of the normal form, even the minimal form of $\widetilde{D}$. This allows to improve significantly the calculation of a class, and to remove the drawbacks that stand in the implementation of other $D B M$ over-approximations.

In the second part of the paper, we propose another abstraction of the state space of an $I T P N$. We show that by relaxing a bit in the precision of the constraints of the system $\widetilde{D}$, we can compute smaller graphs with a minimal cost. However, although this abstraction is less precise than the former, it preserves all the firing sequences of the model and may be sufficient to model check the properties of the $I T P N$. To improve once more this construction, we provide a suitable equivalence relation that contracts the size of the resulted graphs while reducing the effort of their computation. For this effect, we show that for specific transitions, the computation of their firing distances can be useless, and we prove that the equality between these distances is not required in the class equivalence test. This result is 
important since it makes it possible to gather in a same node unequal classes that are indeed bisimilar. This contraction leads to an efficient construction of $D B M$ approximated graphs that can be in certain cases more appropriate to use to model check the linear properties of the model. Moreover, the experiments show that both constructions are faster in all cases while providing, in general, smaller graphs than other fellow approaches [15][7].

The remainder of this paper is organized as follows: In Section 2 we present the syntax and the formal semantics of the ITPN model. Then, in Section 3 we introduce formally our approach. In Section 4 we define a new construction of an abstraction of the state space of an ITPN. In Section 5 we give some experimental results that compare the performances of our algorithms with those of other approaches.

\section{Time Petri Net with Inhibitor Arcs}

Time Petri nets with inhibitor arcs (ITPN) [15] extend time Petri nets [9] with Stopwatch inhibitor arcs. Formally, an ITPN is defined as follows:

Definition 1. An ITPN is given by the tuple $\left(P, T, B, F, M^{0}, I, I H\right)$ where: $P$ and $T$ are respectively two nonempty sets of places and transitions; $B$ is the backward function ${ }^{1}: B: P \times T \longrightarrow \mathbb{N}=\{0,1,2, .\} ;$.$F is the forward function F: P \times T \longrightarrow$ $\mathbb{N} ; M^{0}$ is the initial marking mapping $M^{0}: P \longrightarrow \mathbb{N} ; I$ is the delay mapping $I: T \longrightarrow \mathbb{Q}^{+} \times \mathbb{Q}^{+} \cup\{\infty\}$, where $\mathbb{Q}^{+}$is set of non negative rational. We write $I(t)=[\operatorname{tmin}(t), \operatorname{tmax}(t)]$ such that $0 \leq t \min (t) \leq t \max (t) ; I H: P \times T \longrightarrow \mathbb{N}$ is the inhibitor arc function; there is an inhibitor arc connecting the place $p$ to the transition $t$, if $\operatorname{IH}(p, t) \neq 0$.

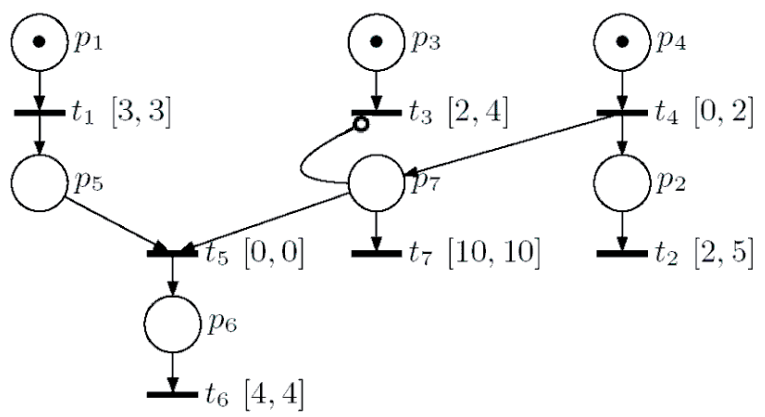

Figure 1: An ITPN model

For instance, let us consider the ITPN model shown in Figure 1, already presented in [15]. Therein, the inhibitor arc is the arc ended by a circle that connects

\footnotetext{
${ }^{1} \mathbb{N}$ denotes the set of positive integers. In the graphical representation, we represent only arcs of non null valuation, and those valued 1 are implicit.
} 
the place $p_{7}$ to the transition $t_{3}$. Initially, the place $p_{3}$ is marked but not the place $p_{7}$; hence $t_{3}$ is enabled but not inhibited. Therefore, $t_{3}$ is progressing, as $t_{4}$ which is also enabled for the initial marking. However, the firing of the transition $t_{4}$ consumes the token in the place $p_{4}$ and produces a new one in $p_{2}$ and another one in $p_{7}$. Therefore, the inhibitor arc connected to $t_{3}$ is activated and hence the clock of $t_{3}$ is suspended ( $t_{3}$ is thus inhibited); this time suspension lasts as long as $p_{7}$ remains marked. For more details, the formal semantics of the ITPN model is introduced in the next section.

Let $R T:=\left(P, T, B, F, M^{0}, I, I H\right)$ be an ITPN.

- We call a marking the mapping, noted $M$, which associates with each place a number of tokens: $M: P \rightarrow \mathbb{N}$.

- A transition $t$ is said to be enabled for the marking $M$, if $\forall p \in P, B(p, t) \leq M(p)$; the number of tokens in each input place of $t$ is greater or equal to the valuation of the arc connecting this place to the transition $t$. Thereafter, we denote by $T e(M)$ the set of transitions enabled for the marking $M$.

- A transition $t$ is said to be inhibited for a marking $M$, if it is enabled and if there exists an inhibitor arc connected to $t$, such that the marking satisfies its valuation $(t \in T e(M)) \wedge \exists p \in P, 0<I H(p, t) \leq M(p)$. We denote by $\operatorname{Ti}(M)$ the set of transitions that are inhibited for the marking $M$.

- A transition $t$ is said to be activated for a marking $M$, if it is enabled and not inhibited, $(t \in T e(M)) \wedge(t \notin T i(M))$; we denote by $T a(M)$ the set of transitions that are activated for the marking $M$.

- Let $M$ be a marking; two transitions $t_{i}$ and $t_{j}$ enabled for $M$ are said to be conflicting for $M$, if $\exists p \in P, \quad B\left(p, t_{i}\right)+B\left(p, t_{j}\right)>M(p)$.

- We note $\operatorname{Conf}(M)$ the relation built on $T e(M)^{2}$ such that $\left(t_{1}, t_{2}\right) \in \operatorname{Conf}(M)$, iff $t_{1}$ and $t_{2}$ are in conflict for the marking $M$.

For instance, let us consider again the $I T P N$ of Figure 1 ; its initial marking is equal to $M^{0}:\left\{p_{1}, p_{3}, p_{4}\right\} \rightarrow 1 ;\left\{p_{2}, p_{5}, p_{6}, p_{7}\right\} \rightarrow 0$; the sets of enabled, inhibited, and activated transitions for $M^{0}$ are respectively $\operatorname{Te}\left(M^{0}\right)=\left\{t_{1}\right\}, \operatorname{Ti}\left(M^{0}\right)=\varnothing$, and $T a\left(M^{0}\right)=T e\left(M^{0}\right)$.

Remark 1. We assume in the sequel a monoserver semantics, which means that for a given marking a transition can be enabled at most once .

We define the semantics of an ITPN as follows:

Definition 2. The semantics of an ITPN is defined as a LTS (labeled transition system $), S T=\left(\Gamma, e^{0}, \rightarrow\right)$, such that: 
- $\Gamma$ is the set of reachable states: Each state, noted e, pertaining to $\Gamma$ is a pair $(M, V)$ where $M$ is a marking and $V$ is a valuation function that associates with each enabled transition $t$ of $\mathrm{Te}(M)$ a time interval that gives the range of relative times within which $t$ can be fired. Formally we have : $\forall t$ $\in T e(M), \quad V(t):=[x(t), y(t)]$

- $e^{0}=\left(M^{0}, V^{0}\right)$ is the initial state, such that: $\forall t \in T e\left(M^{0}\right), \quad V^{0}(t):=I(t):=$ $[\operatorname{tmin}(t), \operatorname{tmax}(t)]$.

- $\rightarrow \in \Gamma \times\left(T \times \mathbb{Q}^{+}\right) \times \Gamma$ is a transition relation, such that

$\left((M, V),\left(t_{f}, t_{f}\right),\left(M^{\uparrow}, V^{\uparrow}\right)\right) \in \rightarrow$ iff:

(i) $t_{f} \in \operatorname{Ta}(M)$.

(ii) $x\left(t_{f}\right) \leq \underline{t_{f}} \leq \underset{\forall t \in \operatorname{Ma}(M)}{\operatorname{MIN}}\{y(t)\}$.

and we have:

$\forall p \in P, M^{\uparrow}(p):=M(p)-B\left(p, t_{f}\right)+F\left(p, t_{f}\right)$.

$\forall t \in T e\left(M^{\uparrow}\right)$

if $t \notin N e w\left(M^{\uparrow}\right)$ :

$\left[x^{\uparrow}(t), y^{\uparrow}(t)\right]:=\left[M A X\left(0, x(t)-t_{f}\right), y(t)-t_{f}\right] \quad t \in T a(M)$

$\left[x^{\uparrow}(t), y^{\uparrow}(t)\right]:=[x(t), y(t)]$

$t \in T i(M)$

if $t \in N e w\left(M^{\uparrow}\right)$

$\left[x^{\uparrow}(t), y^{\uparrow}(t)\right]:=I(t)=[\operatorname{tmin}(t), \operatorname{tmax}(t)]$

- where New $\left(M^{\uparrow}\right)$ denotes the set of transitions newly enabled for the marking $M^{\uparrow}$. These transitions are those enabled for $M^{\uparrow}$ and not for $M$, or those enabled for $M^{\uparrow}$ and $M$ but are conflicting with $t_{f}$ for the marking $M$. Otherwise, an enabled transition which does not belong to $\operatorname{New}\left(M^{\uparrow}\right)$ is said to be persistent.

If $t$ is an enabled transition for a state $e$, we note $\underline{t}$ the clock associated with $t$ that takes its values in $\mathbb{Q}^{+} . \underline{t}$ measures the residual time of the transition $t$ relatively to the instant where the state $e$ is reached. The time progresses only for activated transitions, whereas it is suspended for inhibited transitions. Therefore, a transition $t_{f}$ can be fired at relative time $t_{f}$ from a reachable state $e$, if $(i) t_{f}$ is activated for the marking $M$, and if $(i i)$ the time can progress within the firing interval of $t_{f}$ while satisfying the time constraints of other activated transitions. After firing $t_{f}$ the reachable state, noted $e^{\uparrow}$, is obtained:

- by consuming a number of tokens in each input place $p$ of $t_{f}$ (given by the value $\left.B\left(p, t_{f}\right)\right)$, and by producing a number of tokens in each output place $p$ of $t_{f}$ (given by the value $F\left(p, t_{f}\right)$ ); 
- by shifting the interval of a persistent activated transition with the value of the firing time of $t_{f}$. However, the intervals of persistent inhibited transitions remain unchanged. Finally, newly enabled transitions are assigned their static firing intervals.

Similarly as for $T P N$, the behavior of an $I T P N$ can be defined as a timed sequence of pairs $\left(t_{f}, \delta\right)$, where $t_{f}$ is a transition of the net and $\delta \in \mathbb{Q}^{+}$. Therefore, the timed sequence $S^{*}=\left(\left(t_{f}^{1}, \delta^{1}\right),\left(t_{f}^{2}, \delta^{2}\right), . .,\left(t_{f}^{n}, \delta^{n}\right)\right)$ denotes that $t_{f}^{1}$ is fired after $t_{f}^{1}=$ $\delta^{1}$ time units, then $t_{f}^{2}$ is fired at relative time $t_{f}^{2}=\delta^{2}$ and so on, such that $t_{f}^{n}$ is fired at relative time $t_{f}^{n}=\delta^{n}$ after an absolute time $\sum_{i=1}^{n} \delta^{i}$. Moreover, we often express the behavior of the net as an untimed sequence, denoted by $S$, obtained from a timed sequence $S^{t}$ by removing the firing times: If $S^{*}=\left(\left(t_{f}^{1}, \delta^{1}\right),\left(t_{f}^{2}, \delta^{2}\right), \ldots,\left(t_{f}^{n}, \delta^{n}\right)\right)$, then $S=\left(t_{f}^{1}, t_{f}^{2}, . ., t_{f}^{n}\right)$. Furthermore, a marking $M$ is said to be reachable in $S T$ if there exists an untimed sequence $S$ in $S T$, going from the initial marking $M^{0}$ towards $M$. As the set of time values is assumed to be dense, the model $S T$ is infinite. In order to analyze this model, we need to compute an abstraction of it that saves the most interesting properties. The symbolic graph construction [12] preserves the untimed sequences of $S T$, and makes it possible to compute a finite graph in almost all cases. However, this contraction might be infinite too when the number of reachable markings is unbounded. As this last property is undecidable for $I T P N[15]$, there is no guarantee to compute a finite graph. We show hereafter how to compute the state class graph of the $\operatorname{ITPN}$ that preserves the linear properties of the model.

\section{$3 \quad I T P N$ state space construction}

For a $T P N[9]$, the state class graph method [4] computes a symbolic graph that preserves mainly the linear properties of the model. Similarly, this construction can be applied to an ITPN. This consists in regrouping in a same class all the states reachable after firing the same untimed sequence of transitions; all the states of a same class have the same marking $M$. Hence, a class is defined by the pair $(M, D)$ where $M$ is the common marking of all the states of the class, and $D$ is a set of inequalities encoding the firing space of the class. $D$ is of a general form, normal form of which is expressed as a conjunction of two subsystems $\vec{D} \wedge \widehat{D}$. Actually, $\vec{D}$ contains only $D B M$ constraints and $\widehat{D}$ contains all other constraints than $D B M$. In the sequel, we refer to $\vec{D}$ as the tightest $D B M$ system that over-approximates the system $D$. More formally, a class is defined as follows:

Definition 3. Let $S T=\left(\Gamma, e^{0}, \rightarrow\right)$ be the LTS associated with an ITPN. A class of states of an ITPN, noted $E$, is the set of all the states pertaining to $\Gamma$ that are reachable after firing the same untimed sequence $S=\left(t_{f}^{1}, . ., t_{f}^{n}\right)$ from the initial state $e^{0}$. A class $E$ is defined by $(M, D)$, where $M$ is the marking reachable after firing $S$, and $D$ is the firing space encoded as a set of inequalities. For $T e(M)=\left\{t_{1}, . ., t_{s}\right\}$, we have : $D=\widehat{D} \wedge \vec{D}$ 


$$
\begin{aligned}
& \vec{D}:=\left\{\begin{array}{l}
\bigwedge_{i \neq j}\left(\underline{t_{j}}-\underline{t_{i}} \leq d_{i j}\right) \\
\bigwedge_{i \leq s}\left(d_{i \bullet} \leq \underline{t_{i}} \leq d_{\bullet i}\right)
\end{array}\right. \\
& \text { with }\left(t_{j}, t_{i}\right) \in T e(M)^{2} \quad d_{i j} \in \mathbb{Q} \cup\{\infty\}, d_{\bullet i} \in \mathbb{Q}^{+} \cup\{\infty\}, d_{i \bullet} \in \mathbb{Q}^{+} \\
& \widehat{D}:=\bigwedge_{k=1 . . p}\left(\alpha_{1 k} \underline{t_{1}}+. .+\alpha_{s k} \underline{t_{s}} \leq d_{k}\right) \\
& \text { with } d_{k} \in \mathbb{Q} \cup\{\infty\},\left(\alpha_{1 k}, . ., \alpha_{s k}\right) \in \mathbb{Z}^{s}, p \in \mathbb{N} \text { and } d^{2} \\
& \forall k, \exists(i, j),\left(\alpha_{i k}, \alpha_{j k}\right) \notin\{(0,0),(0,1),(0,-1),(1,-1)\}
\end{aligned}
$$

We denote by the element $\{\bullet\}$ the instant at which the class $E$ is reached. Therefore, the value of the clock $\underline{t_{i}}$ expresses the time relative to the instant $\bullet$, at which the transition $t_{i}$ can be fired. To each valuation $\psi$ satisfying the system $D$, corresponds a unique state $e=(M, V)$ reachable in $S T$ after firing the sequence $S$.

In case of a $T P N$, the system $D$ is reduced to the subsystem $\vec{D}$. The inequalities of the latter have a particular form, called DBM (Difference Bound Matrix)[8]. The coefficients, $d_{\bullet i}, d_{i \bullet}$ and $d_{i j}$ are respectively, the minimum residual time to fire the transition $t_{i}$, the maximum residual time to fire the transition $t_{i}$, and the maximal firing distance of the transition $t_{i}$ relatively to $t_{j}$. It should be noticed that the value of $d_{\bullet i}$ and $d_{i \bullet}$ are always positive or null, whereas the value of $d_{i j}$ can be negative, thus denoting that there exists no state $e$ reachable in $E$, such that $t_{i}$ can be firable from $e$.

For a $T P N$, the firing space of a class can be always encoded as a $D B M$ system. This form makes it possible to apply an efficient algorithm to compute the reachable class from a class $E$. The overall complexity of this algorithm is $O\left(m^{3}\right)$, where $m$ is the number of enabled transitions in $E$. However, for a TPN augmented with stopwatches, the state space of a class may require polyhedra to be encoded, manipulation of which induces an exponential complexity in the worst case. The exact state class graph of an ITPN is computed by enumerating and exploring all the classes reachable from the initial class $E^{0}$. However, as the number of reachable classes may be unbounded, the termination of the algorithm is undecidable. Formally, the exact state class graph of an ITPN can be defined as follows [5]:

Definition 4. The exact state class graph of an ITPN, denoted by $G R$, is the tuple $\left(C E, E^{0}, \longmapsto\right)$ where:

- $C E$ is the set of classes reachable in GR;

- $E^{0}=\left(M^{0}, D^{0}\right)$ is the initial class; $D^{0}=\left\{\forall t_{i} \in T e\left(M^{0}\right), \quad \operatorname{tmin}\left(t_{i}\right) \leq \underline{t_{i}} \leq \operatorname{tmax}\left(t_{i}\right)\right\}$

- $\longmapsto$ is the transition relation between classes defined on $C E \times T \times C E$, such that $\left((M, D), t_{f},\left(M^{\uparrow}, D^{\uparrow}\right)\right) \in \longmapsto$, iff:

a) $t_{f}$ is activated and the system $D$ augmented with the firing constraints of $t_{f}$ that we write $D_{a}=D \wedge\left(\forall t \in T a(M), \quad \underline{t_{f}} \leq \underline{t}\right)$ holds.

\footnotetext{
${ }^{2} \mathbb{Z}$ denotes the set of relative integers.
} 
b) $\forall p \in P, M^{\uparrow}(p):=M(p)-B\left(p, t_{f}\right)+F\left(p, t_{f}\right)$.

c) The system $D^{\uparrow}$ is computed from $D$, as follows:

1. In the system $D_{a}$, replace each variable $\underline{t}$ (related to an enabled transition that is not inhibited for $M)$, by: $\underline{t}:=t_{f}+\underline{t}^{\prime}$, thus denoting the time progression.

2. Eliminate then by substitution the variable $t_{f}$ as well as all the variables relative to transitions disabled by the firing of $t_{f}$;

3. Add to the system thus computed, the time constraints relative to each newly enabled transition for $M^{\uparrow}$ :

$\forall t_{i} \in \operatorname{New}\left(M^{\uparrow}\right), \quad \operatorname{tmin}\left(t_{i}\right) \leq \underline{t_{i}} \leq \operatorname{tmax}\left(t_{i}\right)$

The last definition shows how the exact state class graph of an ITPN is built. Being given a class $E=(M, D)$ and a transition $t_{f}$ activated for $M$, the computation of a class $E^{\uparrow}=\left(M^{\uparrow}, D^{\uparrow}\right)$ reachable from $E$ by firing $t_{f}$ consists in computing the reachable marking $M^{\uparrow}$ and the firing space induced by the new system $D^{\uparrow}$. The class $E$ can fire the transition $t_{f}$, if there exists a valuation that satisfies $D$ (a state of $E$ ), such that $t_{f}$ can be fired before all the other activated transitions. The firing of $t_{f}$ produces a new class $E^{\uparrow}=\left(M^{\uparrow}, D^{\uparrow}\right)$ which gathers all the states reachable from those of $E$. The system $D^{\uparrow}$ that encodes the space of $E^{\uparrow}$ is computed from the system $D$ augmented with the firing constraints of $t_{f}$. The substitution of variables relative to activated transitions allows shifting the time origin to the instant at which the new class $E^{\uparrow}$ is reached. Then, a new system is computed wherein the variables of transitions disabled following the firing of $t_{f}$ are removed. Finally, the time constraints relative to newly enabled transitions are added.

The complexity of the firing test and the step 2 of the algorithm, depends on the form of the system $D$. If $D$ includes polyhedral constraints, then the complexity of the algorithm is exponential, otherwise it is polynomial. The initial system $D^{0}$ is always in $D B M$ form, and polyhedral constraints may appear in reachable classes only when inhibited and activated transitions are both persistently enabled in a firing sequence [7].

Knowing how to compute the successors of a class, the state class graph computation is based on a depth-first or breadth-first strategy. Then the state class graph is given as the quotient of $G R$ by a suitable equivalence relation. This equivalence relation may be equality: two classes $(M, D)$ and $\left(M, D^{\prime}\right)$ given in their minimal form are equal if $D=D^{\prime}$, or inclusion; in other terms, if $\rceil D\lceil$ denotes the set of solutions for the system $D$, then we have : $\rceil D\lceil\subseteq\rceil D^{\prime}\lceil$. It should be noticed that the equality preserves mainly the untimed language of the model, whereas the inclusion preserves the set of reachable markings. In order to speed up the class' equivalence test, the reachable systems are computed in their minimal form; this implies that all redundant inequalities are removed. Moreover, it is proved, as for $D B M$ systems [4], that the minimal form of a polyhedral system is unique [1]. This property is very important as it permits to detect equivalent classes by comparing their minimal form. 
The algorithm given in Definition 4 can be applied to a TPN with the particularity that the system $D$ is always encoded in $D B M s$. Besides, Berthomieu et al proved that the number of equivalent $D B M$ systems computed for a $T P N$ is finite [3]. This implies that the resulted graph is necessarily finite, if the number of reachable markings is bounded.

For $T P N$ augmented with stopwatches, the $D B M$ over-approximation technique has been proposed as an alternative solution to analyze preemptive real time systems [15][7]. This approach consists in cutting off the inequalities of the subsystem $\widehat{D}$ when they are generated in $D$. It thereby keeps only those of the subsystem $\vec{D}$ to represent an over-approximation of the space of $D$. This solution makes it possible to build an approximated graph with lesser expenses in terms of computation time and memory usage. In addition, the $D B M$ over-approximation ensures that the number of computed $D B M$ systems is always finite, whereas that of polyhedral systems may be infinite. Therefore, we can compute a finite approximated graph when the computation of the exact graph does not terminate. However, the $D B M$ over-approximation may compute an infinity of unreachable markings while the exact construction is indeed bounded. For a better understanding of how this technique works, we apply the state class graph method to the ITPN example of Figure 1. Let $E^{\prime}=\left(M^{\prime}, D^{\prime}\right)$ be the class reachable in the exact graph after firing the sequence $S=\left(t_{4}, t_{1}, t_{5}\right)$ from the initial class $E^{0}=\left(M^{0}, D^{0}\right)$.

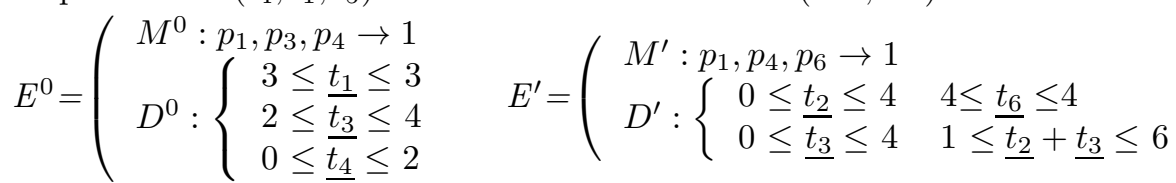

We notice that the transition $t_{6}$ is not firable from $E^{\prime}$ since $t_{2}$ or $t_{3}$ should be fired before. Put in other way, the firing of $t_{6}$ requires that the system $D^{\prime} \wedge\left(\underline{t}_{6} \leq\right.$ $\left.\underline{t_{3}}\right) \wedge\left(\underline{t_{6}} \leq \underline{t_{2}}\right)$ admits at least one solution; we should check whether $\left(\underline{t_{2}}=\underline{t_{3}}=\underline{t_{6}}=\right.$ $\overline{4)} \wedge\left(\overline{t_{2}}+\underline{t_{3}} \leq 6\right)$ holds, or not. As this last inequality is not satisfied, therefore $t_{6}$ cannot fire. The system $D^{\prime}$ contains a polyhedral constraint that cannot be reduced to a $D B M$. The $D B M$ over-approximation consists in cutting off the polyhedron $1 \leq t_{2}+t_{3} \leq 6$ to leave only $D B M$ constraints to represent the state space of $E^{\prime}$. However, by doing this, $t_{6}$ becomes firable since $\underline{t_{6}}=4$ holds. Therefore, the system $\widetilde{D^{\prime}}=\overrightarrow{D^{\prime}}$ denotes an over-approximation of the system $D^{\prime}$. In other words, we add new states in the class $E^{\prime}$ that are not reachable indeed. Nevertheless, this construction makes it possible to preserve a subset of properties.

The computation of the tightest $D B M$ over-approximation of a class $E$ can be obtained by applying different algorithms [15][7]. To reduce the memory usage and to ease the equivalence test, the previous algorithms compute the $D B M$ system of each class in its minimal form. These approaches proceed first to compute the polyhedra, then eliminate the non $D B M$ constraints while normalizing the remaining ones. Then the process terminates by computing the final system in its minimal form.

However, the implementation of the algorithm defined [15] in ROMEO [17] has revealed a loss in the precision of the $D B M$ approximation. This is due to some 
improvements in the computation of normalized systems. On the other side, the computation of the graphs in ORIS [16] (which implements the approach defined [7]), reports very slow times although the resulted graphs are correct.

We show in the sequel that by maintaining all the $D B M$ constraints, even the redundant ones, we succeed to compute the tightest $D B M$ approximated class in $o\left(m^{2}\right)$. In concrete terms, we show that by avoiding to calculate the minimal form, we succeed to define an algorithm that computes straightforwardly a normalized $D B M$ system. We thereby eliminate the computation and the manipulation of the intermediary polyhedra that stand in the other algorithms. Moreover, we improve greatly the implementation of the graph construction and remove the bugs reported in $R O M E O$.

Formally, a DBM over-approximated class of an ITPN can be defined as follows:

Definition 5. (Approximated Class). A DBM over-approximated class of an ITPN, noted $\widetilde{E}$, is the pair $(M, \widetilde{D})$ such that : $M$ is a marking and $\widetilde{D}$ is the full system of all DBM normalized inequalities, involving all variables of transitions enabled for $M$ :

$$
\begin{aligned}
\widetilde{D}= & \left\{\begin{array}{l}
\bigwedge_{\forall\left(t_{i}, t_{j}\right) \in T e(M)^{2}} \quad\left(\underline{t_{j}}-\underline{t_{i}} \leq \widetilde{d_{i j}}\right) \\
\bigwedge_{\forall t_{i} \in T e(M)}\left(\widetilde{d_{i}} \leq \underline{t_{i}} \leq \widetilde{d_{\bullet i}}\right)
\end{array}\right. \\
& \text { with }\left(t_{j} \neq t_{i}\right), \widetilde{d_{i j}} \in \mathbb{Q} \cup\{\infty\}, \widetilde{d_{\bullet i}} \in \mathbb{Q}^{+} \cup\{\infty\}, \widetilde{d_{i}} \in \mathbb{Q}^{+}:
\end{aligned}
$$

such that each inequality is in the normal form:

$\forall x, y, z \in T e(M),\left(\widetilde{d_{x y}} \leq \widetilde{d_{x z}}+\widetilde{d_{z y}}\right) \wedge\left(\widetilde{d_{x y}} \leq \widetilde{d_{\bullet y}}-\widetilde{d_{x} \bullet}\right)$

The space of a $D B M$ over-approximated class is encoded by the system $\widetilde{D}$. Besides, we assume that the system $\widetilde{D}$ is given in its normal form. As for the minimal form ${ }^{3}$, it is proved that this form is unique for a $D B M$ system [7] ; all equivalent systems have the same normal form.

In the sequel, we encode the system $\widetilde{D}$ as a square matrix where each line and corresponding column, are indexed by an element of $T e(M) \cup\{\bullet\}$. In concrete terms, we have:

$$
\begin{gathered}
\forall\left(t_{i}, t_{j}\right) \in T e(M)^{2} \wedge\left(t_{i} \neq t_{j}\right), \quad \widetilde{D}\left[\bullet, t_{i}\right]:=\widetilde{d_{\bullet i}} ; \quad \widetilde{D}\left[t_{i}, \bullet\right]:=-\widetilde{d_{i}} ; \\
\widetilde{D}\left[t_{i}, t_{j}\right]:=\widetilde{d_{i j}} ; \quad \widetilde{D}\left[t_{i}, t_{i}\right]:=0 ; \quad \widetilde{D}[\bullet, \bullet]:=0 .
\end{gathered}
$$

These matrix notations are used to represent the coefficients of the system $\widetilde{D}$. For example, the matrix shown in Table 1 encodes the system $\widetilde{D^{0}}=D^{0}$ associated with the initial class of the exact graph of the ITPN of Figure 1. It is noteworthy that the approximated class $\widetilde{E^{0}}=\left(M^{0}, \widetilde{D^{0}}\right)$ is in the normal form, and represents an exact over-approximation of the initial class $E^{0}=\left(M^{0}, D^{0}\right)$ of the graph $G R$. The minimal form of the system $\widetilde{D^{0}}$ is given by: $\left(\underline{t_{1}}=3\right) \wedge\left(2 \leq \underline{t_{3}} \leq 4\right) \wedge\left(0 \leq \underline{t_{4}} \leq 2\right)$.

\footnotetext{
${ }^{3}$ The minimal form of a $D B M$ system is obtained from its normal form by cutting off all redundant inequalities.
} 
Table 1: The matrix representation of the system $\widetilde{D^{0}}$.

\begin{tabular}{|l||l|l|l|l|}
\hline$D^{0}$ & $\bullet$ & $t_{1}$ & $t_{3}$ & $t_{4}$ \\
\hline \hline$\bullet$ & 0 & 3 & 4 & 2 \\
\hline$t_{1}$ & -3 & 0 & 1 & -1 \\
\hline$t_{3}$ & -2 & 1 & 0 & 0 \\
\hline$t_{4}$ & 0 & 3 & 4 & 0 \\
\hline
\end{tabular}

Taking on the previous definition, if $E=(M, D)$ is a class reachable in $G R$, then the class $\widetilde{E}=(M, \widetilde{D})$ is an over-approximation of $E$, if the space of states of $E$ is included in that of $\widetilde{E}$, and we have: $\rceil D\lceil\subseteq\rceil \widetilde{D}\lceil$. Hence, by substituting $\widetilde{E}$ for $E$ in the graph $G R$, it results that the class $\widetilde{E}$ may derive additional sequences that are not firable from $E$ in $G R$. We thereby obtain an over-approximation of the graph $G R$, that we build as defined next:

Definition 6. The graph of DBM over-approximated classes of an ITPN, denoted by $\widetilde{G R}$, is the tuple $\left(\widetilde{C E}, \widetilde{E^{0}}, \rightsquigarrow\right)$, such that:

- $\widetilde{C E}$ is the set of approximated classes reachable in $\widetilde{G R}$;

- $\widetilde{E^{0}}=\left(M^{0}, \widetilde{D^{0}}\right) \in \widetilde{C E}$ is the initial class, such that:

$$
\widetilde{D^{0}}:= \begin{cases}\forall t_{i} \in T e\left(M^{0}\right), & \operatorname{tmin}\left(t_{i}\right) \leq \underline{t_{i}} \leq \operatorname{tmax}\left(t_{i}\right) \\ \forall t_{i} \neq t_{j} \in T e\left(M^{0}\right), \quad \underline{t_{j}}-\underline{t_{i}} \leq \operatorname{tmax}\left(t_{j}\right)-\operatorname{tmin}\left(t_{i}\right)\end{cases}
$$

- $\rightsquigarrow$ is a transition relation between approximated classes defined on $\widetilde{C E} \times T \times$ $\widetilde{C E}$, such that $\left((M, \widetilde{D}), t_{f},\left(M^{\uparrow}, \widetilde{D^{\uparrow}}\right)\right) \in \rightsquigarrow$, iff :

$-\left(t_{f} \in T a(M)\right) \wedge\left(\widetilde{\beta}\left[t_{f}\right] \geq 0\right)$ such that: $\forall x \in T e(M) \cup\{\bullet\}, \quad \widetilde{\beta}[x]=$ $\underset{\forall t \in T a(M)}{\operatorname{MIN}}\{\widetilde{D}[x, t]\}$.

$-\forall p \in P, M^{\uparrow}(p):=M(p)-B\left(p, t_{f}\right)+F\left(p, t_{f}\right)$.

- The coefficients of the DBM inequalities of the system $\widetilde{D^{\uparrow}}$ are computed from those of $\widetilde{D}$ by applying the following algorithm:

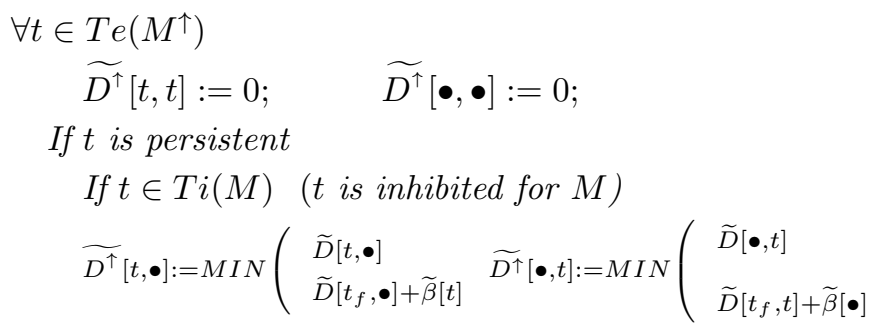




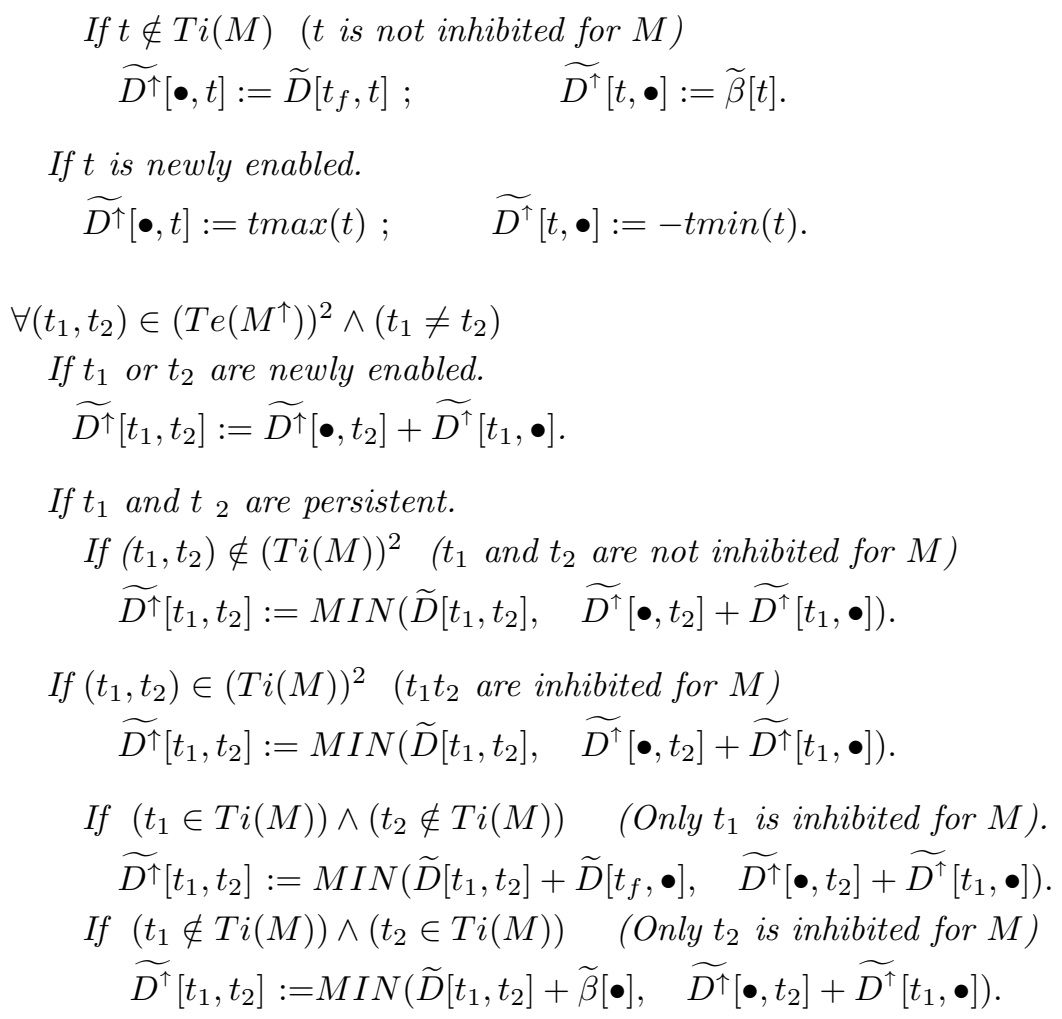

If $t$ is an activated transition, then $\widetilde{\beta}[t]$ denotes the minimal time distance between its firing time and any other firable transition. Further, $\widetilde{\beta}[\bullet]$ represents the maximal dwelling time in the class $\widetilde{E}$. Therefore, an activated transition $t_{f}$ is not firable from $\widetilde{E}$, if $\widetilde{\beta}\left[t_{f}\right]<0$. In other words, it does not exist any state reachable in $\widetilde{E}$ such that the valuation of the clock associated with $t_{f}$ can overtake the minimal bound $\operatorname{tmin}\left(t_{f}\right)$. For a better understanding, the Figure 2.a. depicts the computation of the coefficients $\widetilde{\beta}\left[t_{a}\right], \widetilde{D^{\uparrow}}\left[\bullet, t_{a}\right]$ and $\widetilde{D^{\uparrow}}\left[t_{a}, \bullet\right]$ for $t_{a} \in T a(M)$.

Moreover, we notice that the maximal residual time of an inhibited transition $t_{h}$ can decrease after firing $t_{f}$. Besides, the minimal residual time of $t_{h}$ can increase. To clarify this point, let us consider the ITPN of Figure 1. Initially $t_{3}$ is activated with $\widetilde{D}\left[t_{3}, \bullet\right]=-2$, and the model can fire the transition $t_{4}$ between $[0,2]$. After this firing, the place $p_{7}$ becomes marked, and $t_{3}$ is inhibited for the first time; we have $\widetilde{D}\left[t_{3}, \bullet\right]=0$. Then, to fire the newly enabled transition $t_{2}$, it needs to let time progress at least with $\operatorname{tmin}\left(t_{2}\right)=2$, while the absolute time must not surpass $\operatorname{tmax}\left(t_{1}\right)=3$. This last constraint restricts the state space of the class reachable after firing $t_{2}$ only to states that have fire initially $t_{4}$ during $[0,1]$. As a result, the minimal residual time of $t_{3}$ increases after the firing of $t_{2}$ (see Figure 2.b)

In other respects, the firing distance $\widetilde{D}\left[t_{a}, t_{h}\right]$ between an activated transition $t_{a}$ and an inhibited transition $t_{h}$ can only increase after firing $t_{f}$, with the maximal 
dwelling time ${ }^{4}$ in $\widetilde{E}$. Also, the distance $\widetilde{D}\left[t_{h}, t_{a}\right]$ can only decrease after firing $t_{f}$ with the the minimal dwelling time ${ }^{5}$ in $\widetilde{E}$.

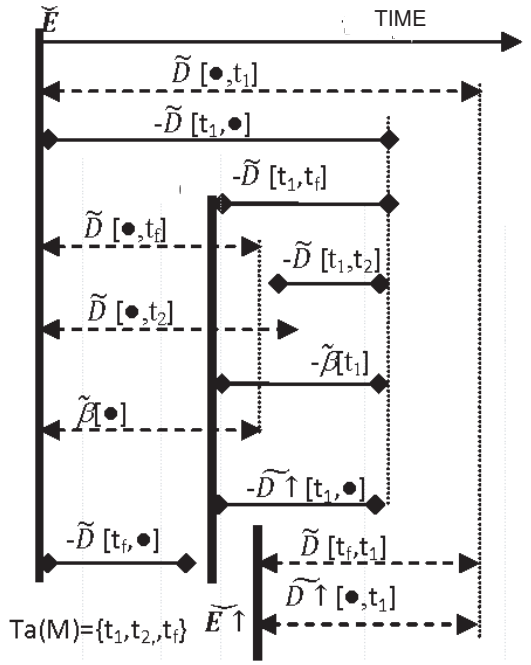

(a)

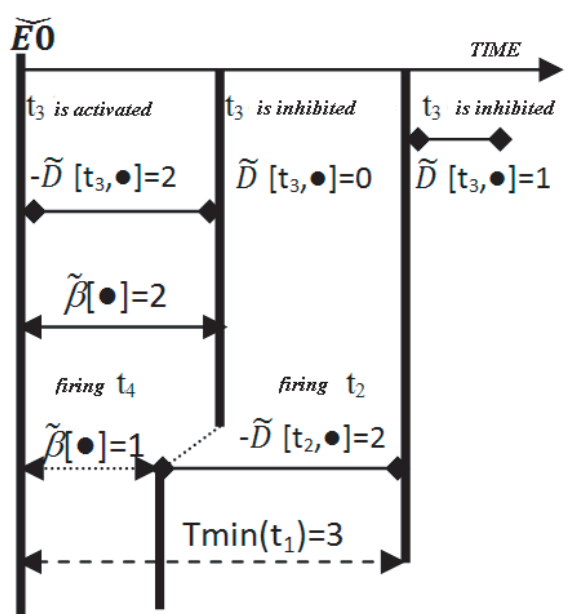

(b)

Figure 2: Computing the $D B M$ coefficients.

It is noteworthy that if $\widetilde{E}$ is an over-approximation of the exact class $E$, then all the transitions firable from $E$ are also firable from $\widetilde{E}$. However, a transition which is not firable from $E$ can, on the other hand, be firable ${ }^{6}$ from $\widetilde{E}$. Actually, as the class $\widetilde{E}$ contains all the states of $E$, we can find at least one state $e$ of $\widetilde{E}$ non reachable in $E$, such that $e$ can fire $t_{f}$. We prove hereafter that the algorithm given in Definition 6 computes in all cases the tightest $D B M$ over-approximation of the exact graph defined in Definition 4 .

Theorem 1. The graph $\widetilde{G R}=\left(\widetilde{C E},\left(M^{0}, \widetilde{D^{0}}\right), \rightsquigarrow\right)$ is the tightest DBM overapproximation that we can compute from the graph $G R=\left(C E,\left(M^{0}, D^{0}\right), \longmapsto\right)$.

Proof. We should prove that:

1. $\rceil D^{0}\lceil\subseteq\rceil \overrightarrow{D^{0}}\lceil=\rceil \widetilde{D^{0}}\lceil$.

2. Let be $S=\left(t_{f}^{1}, . ., t_{f}^{n}\right)$; if $\left(M^{0}, D^{0}\right) \stackrel{t_{f}^{1}}{\longmapsto} . . \stackrel{t_{f}^{n}}{\longmapsto} E=(M, D)$ and $\left(M^{0}, \widetilde{D^{0}}\right) \stackrel{t_{f}^{1}}{\rightsquigarrow}$ $. . \stackrel{t_{f}^{n}}{\rightsquigarrow} \widetilde{E}=(M, \widetilde{D})$, such that $\rceil D\lceil\subseteq\rceil \vec{D}\lceil=\rceil \widetilde{D}\left\lceil\right.$; we have, if $E \stackrel{t_{f}}{\longmapsto} E^{\uparrow}=$

\footnotetext{
${ }^{4}$ This time denotes the maximal time that has elapsed for $t_{a}$ and during which $t_{h}$ has remained suspended.

${ }^{5}$ This time denotes the minimal time that has elapsed for $t_{a}$ and during which $t_{h}$ has remained suspended.

${ }^{6}$ Conversely, if $t_{f}$ is not firable from $\widetilde{E}$, then it is not firable from $E$.
} 


$$
\left.\left.\left.\left(M^{\uparrow}, D^{\uparrow}\right) \text {, then } \widetilde{E} \stackrel{t_{f}}{\rightsquigarrow} \widetilde{E^{\uparrow}}=\left(M^{\uparrow}, \widetilde{D^{\uparrow}}\right) \text { and }\right\rceil D^{\uparrow}\lceil\subseteq\rceil \overrightarrow{D^{\uparrow}}\right\rceil=\right\rceil \widetilde{D^{\uparrow}}\lceil\text {. }
$$

The clause (1) holds since the system $D^{0}$ is in $D B M$; we have by definition : \rceil $D^{0}\lceil=] \overrightarrow{D^{0}}\left\lceil=7 \widetilde{D^{0}}[\right.$. Let us prove now the clause (2). For this effect, we write: $t_{f}$ the transition to fire, $t_{h}$ an inhibited transition of $T i(M)$, and $t_{a}$ an activated transition of $T a(M)-\left\{t_{f}\right\}$. The system $D$ stands as $D=\vec{D} \wedge \widehat{D}$, and wherein we suppose that the system $\vec{D}$ is the full system of all $D B M$ normalized inequalities, given as follows:

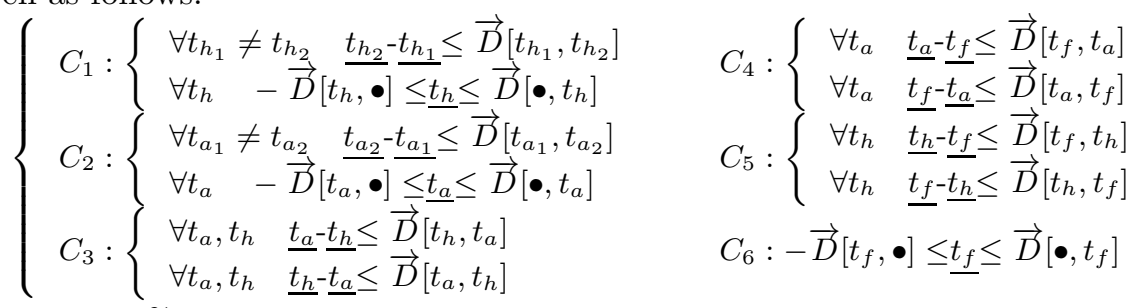

Besides, $\widetilde{D}$ is the tightest $D B M$ over-approximation of $D$; hence the next property holds $(P):] \widetilde{D}\lceil=\rceil \vec{D}\lceil$.

Let us consider now the firing of the transition $t_{f}$ from $E$ to reach the class $E^{\uparrow}=\left(M^{\uparrow}, D^{\uparrow}\right)$. The calculation of the system $D^{\uparrow}$ is performed by application of the algorithm given in Definition 4 . We extend $D$ to the firing constraints of $t_{f}$ , $C_{7}: \forall t_{a} \quad \underline{t}_{a}-t_{f} \geq 0$.

Therefore, if $\overline{t_{f}}$ is firable from $E$, then the system $\rceil D \wedge C_{7}\lceil\neq \emptyset$, and we have the coefficients $\vec{D}\left[t_{f}, t_{a}\right] \geq 0$ and by using the property $(P)$, we deduce that $\widetilde{D}\left[t_{f}, t_{a}\right] \geq$ 0 , hence $\widetilde{\beta}\left[t_{f}\right] \geq 0$. Consequently, $t_{f}$ is also firable from the class $\widetilde{E}$. However, it remains to prove that $\rceil \overrightarrow{D^{\uparrow}}\lceil=\rceil \widetilde{D^{\uparrow}}\lceil$.

The computation of the system $D^{\uparrow}$ is performed by replacing each variable $t_{a}$ associated with an activated transition $t_{a} \in T a(M)-\left\{t_{f}\right\}$ by $\underline{t}_{a}^{\prime}+\underline{t_{f}}$. To ease the sketch of the proof, we suppose that all transitions of $\operatorname{Ti}\left(M^{\uparrow}\right) \cup \bar{T} a\left(\bar{M}^{\uparrow}\right)-\left\{t_{f}\right\}$ are persistent after firing $t_{f}$. Further, we limit the proof to the manipulation of $D B M$ constraints, since we aim at computing the tightest $D B M$ over-approximation that can be derived from $D$ subsequently to the firing of $t_{f}$. It should be noticed that the manipulation of the constraints $\widehat{D}$ produces only new polyhedral constraints that cannot be reduced to $D B M s$ [7]. So, after substitution, the constraints of the subsystem $\vec{D} \wedge C_{7}$ are as follows:

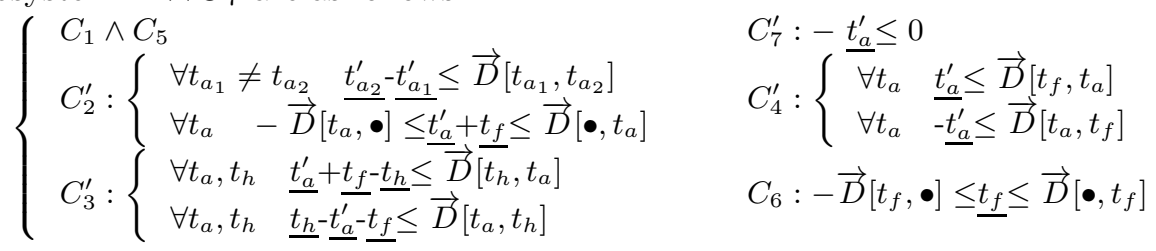

By operating an intersection of the constraints $C_{7}^{\prime}$ and $C_{2}^{\prime}$, we obtain the system: 


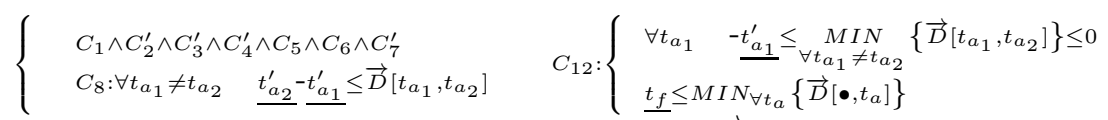

Then by intersection and using the property $\vec{D}\left[t_{a}, t_{a}\right]=0$, the constraints of $C_{12}, C_{6} C_{4}^{\prime}$ change into $C_{6}^{\prime}$ and $C_{4}^{\prime \prime}$; we obtain:

$$
\begin{aligned}
& \left\{\begin{array}{l}
C_{1} \wedge C_{2}^{\prime} \wedge C_{3}^{\prime} \wedge C_{5} \wedge C_{7}^{\prime} \wedge C_{8} \\
C_{6}^{\prime}:-\vec{D}\left[t_{f}, \bullet \leq \underline{t_{f}} \leq \underset{\forall I \in T a(M)}{M I N}\{\vec{D}[\bullet, t]\}\right. \\
C_{4}^{\prime \prime}: \forall t_{a}-\underset{\forall t \in T a(M)}{\operatorname{MIN}} \vec{D}\left[t_{a}, t\right] \leq \underline{t_{a}^{\prime}} \leq \vec{D}\left[t_{f}, t_{a}\right]
\end{array}\right. \\
& \text { We write: }\left\{\begin{array}{c}
\left(F_{1}\right): \forall t_{a} \quad \overrightarrow{D^{\uparrow}}\left[\bullet, t_{a}\right]:=\vec{D}\left[t_{f}, t_{a}\right] \\
\left(F_{2}\right): \overrightarrow{D^{\uparrow}}\left[t_{a}, \bullet\right]:=\underset{\forall t \in T a(M)}{\operatorname{MIN}}\left\{\vec{D}\left[t_{a}, t\right]\right\} .
\end{array}\right.
\end{aligned}
$$

Then by using $C_{8}$ and $C_{4}^{\prime \prime}$, we obtain:

$$
\left\{\begin{array}{l}
C_{1} \wedge C_{2}^{\prime} \wedge C_{3}^{\prime} \wedge C_{4}^{\prime \prime} \wedge C_{5} \wedge C_{6}^{\prime} \wedge C_{7}^{\prime} \\
C_{8}^{\prime}: \forall t_{a_{1}} \neq t_{a_{2}} \quad \underline{t_{a_{2}}^{\prime}} \underline{\underline{t_{a_{1}}^{\prime}}} \leq \operatorname{MIN}\left(\vec{D}\left[t_{a_{1}}, t_{a_{2}}\right], \overrightarrow{D^{\uparrow}}\left[\bullet, t_{a_{2}}\right]+\overrightarrow{D^{\uparrow}}\left[t_{a_{1}}, \bullet\right]\right)
\end{array}\right.
$$

We put

$$
\left(F_{3}\right): \forall t_{a_{1}} \neq t_{a_{2}} \quad \overrightarrow{D^{\uparrow}}\left[t_{a_{1}}, t_{a_{2}}\right]:=\operatorname{MIN}\left(\vec{D}\left[t_{a_{1}}, t_{a_{2}}\right], \overrightarrow{D^{\uparrow}}\left[\bullet, t_{a_{2}}\right]+\overrightarrow{D^{\uparrow}}\left[t_{a_{1}}, \bullet\right]\right) .
$$

At this stage, when the model does not contain inhibitors arcs, the system $D$ stands as $\vec{D}$, and the constraints $C_{1} \wedge C_{3}^{\prime} \wedge C_{5}$ are eliminated and those of $C_{2}^{\prime} \wedge C_{6}^{\prime} \wedge C_{7}^{\prime}$ can be removed as they are redundant. Therefore, the new system $D^{\uparrow}$ is given by the constraints $C_{4}^{\prime \prime} \wedge C_{8}^{\prime}$, to which we add the constraints of newly enabled transitions. The system $\widetilde{D^{\uparrow}}$ obtained from the system $\widetilde{D}$ after firing $t_{f}$ can be computed in the same way as shown previously. Assuming that, if we have $D=\widetilde{D}$, then the algorithm given in Definition 6 computes an exact approximation of the system $D$, since the coefficients $\widetilde{D^{\uparrow}}\left[t_{a_{1}}, t_{a_{2}}\right] \widetilde{D^{\uparrow}}\left[\bullet, t_{a}\right]$ and $\widetilde{D^{\uparrow}}\left[t_{a}, \bullet\right]$ are computed also by using respectively the formulae $F_{3}, F_{1}$ and $F_{2}$.

On the other hand, in presence of inhibited transitions, we need to operate additional manipulations on constraints $C_{1}, C_{2}^{\prime}, C_{3}^{\prime}$ and $C_{5}$ :

By intersection of the constraints of $C_{6}^{\prime}$ and those of $C_{5}$, of $C_{3}^{\prime}$ and those of $C_{7}^{\prime}$, and finally, of $C_{3}^{\prime}$ and those of $C_{6}^{\prime}$; we obtain respectively the new constraints $C_{5}^{\prime}$, $C_{9}$ and $C_{10}$ :

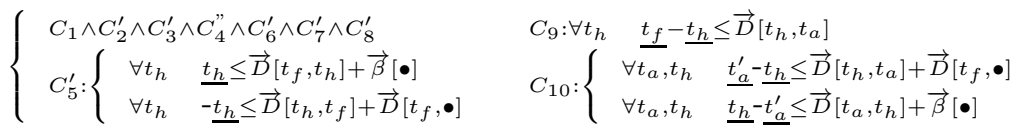

$$
\begin{aligned}
& \text { with } \forall x \in T e(M) \cup\{\bullet\}, \vec{\beta}[x]=\underset{\forall t \in T a(M)}{\operatorname{MIN}}\{\vec{D}[x, t]\}
\end{aligned}
$$

Then by intersection of the constraints of $C_{9}$ with those of $C_{6}^{\prime}$, we obtain the constraints $C_{9}^{\prime \prime}$ :

$$
\left\{\begin{array}{l}
C_{1} \wedge C_{2}^{\prime} \wedge C_{3}^{\prime} \wedge C_{4}^{\prime \prime} \wedge C_{5}^{\prime} \wedge C_{6}^{\prime} \wedge C_{7}^{\prime} \wedge C_{8}^{\prime} \wedge C_{10} \\
C_{9}^{\prime \prime}: \forall t_{h}, \quad-\underline{t_{h}} \leq \vec{D}\left[t_{h}, t_{a}\right]+\vec{D}\left[t_{f}, \bullet\right]
\end{array}\right.
$$

By using the constraints of $C_{9}^{\prime \prime}, C_{5}^{\prime}$ and $C_{1}$ we obtain: 


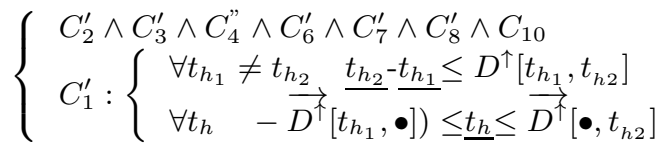

with

$$
\begin{aligned}
& F_{4}: \forall t_{h}, \quad \overrightarrow{D^{\uparrow}}\left[t_{h}, \bullet\right]:=\operatorname{MIN}\left(\begin{array}{l}
\vec{D}\left[t_{h}, \bullet\right] \\
\vec{D}\left[t_{f}, \bullet\right]+\vec{\beta}\left[t_{h}\right]
\end{array}\right. \\
& F_{5}: \forall t_{h}, \quad \overrightarrow{D^{\uparrow}}\left[\bullet, t_{h}\right]:=\operatorname{MIN}\left(\begin{array}{l}
\vec{D}\left[\bullet, t_{h}\right] \\
\vec{D}\left[t_{f}, t_{h}\right]+\vec{\beta}[\bullet]
\end{array}\right. \\
& F_{6}: \forall t_{h_{1}} \neq t_{h_{2}} \quad \overrightarrow{D^{\uparrow}}\left[t_{h_{1}}, t_{h_{2}}\right]:=\operatorname{MIN}\left(\vec{D}\left[t_{h_{1}}, t_{h_{2}}\right], \overrightarrow{D^{\uparrow}}\left[\bullet, t_{h_{2}}\right]+\overrightarrow{D^{\uparrow}}\left[t_{h_{1}}, \bullet\right]\right) .
\end{aligned}
$$

To achieve the proof, we proceed to the intersection of the constraints of $C_{10}$ with those of $C_{1}^{\prime}$ and $C_{4}^{\prime \prime}$; we obtain the constraints $C_{10}^{\prime}$ :

$$
\begin{aligned}
& \left\{\begin{array}{l}
C_{1}^{\prime} \wedge C_{2}^{\prime} \wedge C_{3}^{\prime} \wedge C_{4}^{\prime \prime} \wedge C_{6}^{\prime} \wedge C_{7}^{\prime} \wedge C_{8}^{\prime} \\
C_{10}^{\prime}:\left\{\begin{array}{l}
\forall t_{a}, t_{h} \\
\forall t_{a}, t_{h}
\end{array} \quad \underline{t_{a}^{\prime}}-\underline{t_{h}}-\underline{t_{h}} \leq \overrightarrow{D^{\dagger}} \leq \overrightarrow{D^{\uparrow}}\left[t_{h}, t_{a}, t_{h}\right]\right.
\end{array}\right. \\
& \left(F_{7}\right): \forall t_{h} \forall t_{a} \quad \overrightarrow{D^{\uparrow}}\left[t_{h}, t_{a}\right]:=\operatorname{MIN}\left(\vec{D}\left[t_{h}, t_{a}\right]+\vec{D}\left[t_{f}, \bullet\right], \quad \overrightarrow{D^{\uparrow}}\left[\bullet, t_{a}\right]+\overrightarrow{D^{\uparrow}}\left[t_{h}, \bullet\right]\right) . \\
& \left(F_{8}\right): \forall t_{h} \forall t_{a} \quad \overrightarrow{D^{\uparrow}}\left[t_{a}, t_{h}\right]:=\operatorname{MIN}\left(\vec{D}\left[t_{a}, t_{h}\right]+\vec{\beta}[\bullet], \quad \overrightarrow{D^{\uparrow}}\left[\bullet, t_{h}\right]+\overrightarrow{D^{\uparrow}}\left[t_{a}, \bullet\right]\right) .
\end{aligned}
$$

The remaining manipulations allow to eliminate the transition $t_{f}$, thereby producing only polyhedral constraints that cannot fit into $D B M s$. These manipulations consists in the intersection of the constraints wherein the variable $t_{f}$ occurs: $C_{2}^{\prime}, C_{3}^{\prime}$ and $C_{6}^{\prime}$. Therefore, the system $\overrightarrow{D^{\uparrow}}$ is by construction the much precise $D B M$ system that we can derive from $\vec{D}$ subsequently to the firing of the transition $t_{f}$. Further, assuming that the same algorithm is used to compute the coefficients of the system $\overrightarrow{D^{\uparrow}}$ as well as those of the system $\widetilde{D^{\uparrow}}$, then it is obvious that $\left.\rceil \widetilde{D^{\uparrow}} \mid=\right] \overrightarrow{D^{\uparrow}}\lceil$; the property $(P)$ holds for the systems $\widetilde{D^{\uparrow}}$ and $\overrightarrow{D^{\uparrow}}$. What is more, by assuming the formulae given previously, we prove that if $\widetilde{D}$ is in its normal form then the system $\widetilde{D^{\uparrow}}$ is also in normal form. Put in other way, as the initial class is in normal form, this guarantees, on a hand, that the $D B M$ over-approximation is the tightest that we can compute from $\widetilde{D}$ subsequently to the firing of $t_{f}$. On the other hand, this implies also that the number of $D B M$ that the algorithm can compute is finite, since all reachable approximated classes of the graph are in normal form [7].

Furthermore, the last algorithm should be provided with class equivalence conditions, in order to put an end to the enumeration process when the net is bounded. These conditions are based generally on the equality of markings and systems, as defined next:

Definition 7. Two classes $\widetilde{E}=(M, \widetilde{D})$ and $\widetilde{E^{\prime}}=\left(M^{\prime}, \widetilde{D^{\prime}}\right)$, reachable in $\widetilde{G R}$ satisfying the following conditions, are equivalent, and we write $\widetilde{E}=\widetilde{E^{\prime}}$ :
(i) $\quad M=M^{\prime}$
(ii) $\forall x, y \in(T e(M) \cup\{\bullet\})^{2} \quad \widetilde{D}[x, y]=\widetilde{D^{\prime}}[x, y]$. 
It should be noticed that the finiteness of the exact state class graph is undecidable even for bounded nets [5]. However, the graph obtained by $D B M$ overapproximation is ensured to be finite when the net is bounded. This makes it possible to compute a finite $D B M$ over-approximation when the exact one does not terminate. The approaches defined in [7][15] admit also that the $D B M$ overapproximation that they compute, are the tightest possible. Nevertheless, these techniques have an additional cost comparatively to our algorithm. Concretely, these approaches proceed first to compute the polyhedra in its normal form (whose representation in memory and manipulation are costly), before removing the non $D B M$ constraints and normalizing the $D B M \mathrm{~s}$. Then the process ends by computing the minimal form of the final $D B M$ system. The normalization and the minimization induce a non neglectable computation effort. This affects the performances of the implementation of the $D B M$ over-approximation.

Furthermore, the implementation in $R O M E O$ [17] of the approach defined in [15] does not compute the tightest $D B M$ over-approximation in much of the cases. In actual fact, $R O M E O$ computes, first, the system $D$ in its minimal form, to apply then the normalization to the set of $D B M$ constraints. This makes it impossible to improve the performances of the tool but at the expenses of the precision of the $D B M$ over-approximation. To highlight this point, let us consider the example of Figure 3 already introduced in [7]. This example models three independent tasks that are conflicting for a common resource (CPU): Two periodic tasks 1 and 3 (of period 50 and 150 time units), and one sporadic task with a minimum and maximum inter-arrival times of $[100,150]$. The task 1 (modeled by the transitions $t_{1}$ and $t_{4}$ ), has a higher priority than that of two other tasks, and the sporadic task has a higher priority than that of the third task. The priorities are modeled by using inhibitor arcs.

So, starting from the initial class, the firing of the sequence $\left(t_{4}, t_{5}, t_{1}, t_{4}, t_{6}, t_{1}, t_{2}\right.$, $\left.t_{4}, t_{5}, t_{1}, t_{3}, t_{4}, t_{6}, t_{1}, t_{2}, t_{4}, t_{5}, t_{1}, t_{4}, t_{1}, t_{3}, t_{4}, t_{6}, t_{2}, t_{5}, t_{1}, t_{4}, t_{1}, t_{4}, t_{1}, t_{3}, t_{4}, t_{2}\right)$ yields the classes $\widetilde{E}, \widetilde{E}$ " and $E$ by using respectively, the algorithm introduced in this paper, the $D B M$ over-approximation implemented in the tool $R O M E O$ and finally the exact approach based on polyhedral representation [13] implemented also in $R O M E O$.

$\widetilde{E}=$

$M: p_{2}, p_{3} \rightarrow 1$
\begin{tabular}{|c|c|c|c|c|c|c|}
\hline$\widetilde{D}$ & - & $t_{1}$ & $t_{2}$ & $t_{3}$ & $t_{5}$ & $t_{6}$ \\
\hline$\bullet$ & 0 & 40 & 150 & 140 & 20 & 28 \\
\hline$t_{1}$ & -18 & 0 & 132 & 100 & 2 & -2 \\
\hline$t_{2}$ & -100 & -60 & 0 & 40 & -80 & -72 \\
\hline$t_{3}$ & -118 & -100 & 32 & 0 & -98 & -102 \\
\hline$t_{5}$ & -18 & 22 & 132 & 122 & 0 & 10 \\
\hline$t_{6}$ & 0 & 20 & 150 & 120 & 20 & 0 \\
\hline
\end{tabular}

$$
E=\widetilde{E^{\prime \prime}}=
$$$$
\left\{\begin{array}{l}
M: p_{2}, p_{3} \rightarrow 1 \\
\widetilde{D^{\prime \prime}}: \\
\begin{cases}18 \leq \underline{t_{1}} \leq 40 & \underline{t_{3}}-\underline{t_{6}} \leq 120 \\
100 \leq \underline{t_{2}} \leq 150 & 0 \leq \underline{t_{6}} \leq 28 \\
118 \leq \underline{t_{3}} \leq 140 & \underline{t_{6}}-\underline{t_{1}} \leq-2 \\
18 \leq \underline{t_{5}} \leq 20 & \underline{t_{6}}-\underline{t_{3}} \leq-102 \\
\underline{t_{1}}-\underline{t_{3}} \leq-100 & \\
\underline{t_{1}}-\underline{t_{6}} \leq 20 & \\
\underline{t_{3}}-\underline{t_{1}} \leq 100 & \end{cases}
\end{array}\right.
$$

We notice at this stage that all the resulted classes induce the same firing space whatever the approach we use. In the obtained classes, only the transition $t_{6}$ is inhibited and its maximal residual time is evaluated to 28 . Now, let us consider the 


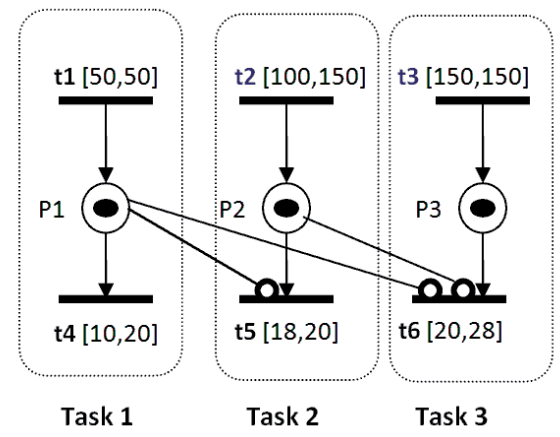

Figure 3: ITPN modeling two periodic tasks and a sporadic one.

firing of the transition $t_{1}$ from the previous classes to reach respectively the classes $\widetilde{E^{1}}, \widetilde{E^{\prime \prime}}$ and $E^{1}$.

$$
\begin{aligned}
& \widetilde{E^{1}}= \\
& \begin{array}{l}
M^{1}: p_{1}, p_{2}, p_{3} \rightarrow 1 \\
\begin{array}{|c|c|c|c|c|c|c|c|}
\hline \widetilde{D}_{1} & \bullet & t_{1} & t_{2} & t_{3} & t_{4} & t_{5} & t_{6} \\
\hline \bullet & 0 & 50 & 132 & 100 & 20 & 2 & 18 \\
\hline t_{1} & -50 & 0 & 82 & 50 & -30 & -48 & -32 \\
\hline t_{2} & -80 & -30 & 0 & 20 & -60 & -80 & -62 \\
\hline t_{3} & -100 & -50 & 32 & 0 & -80 & -98 & -82 \\
\hline t_{4} & -10 & 40 & 122 & 90 & 0 & -8 & 8 \\
\hline t_{5} & 0 & 50 & 132 & 100 & 20 & 0 & 18 \\
\hline t_{6} & 0 & 50 & 132 & 100 & 20 & 2 & 0 \\
\hline
\end{array}
\end{array} \\
& \widetilde{E^{\prime 1}}=\left(\widetilde{M^{1}: p_{1}, p_{2}, p_{3} \rightarrow 1} \begin{array}{l}
\widetilde{D^{\prime 1}}: \begin{cases}50 \leq \underline{t_{1}} \leq 50 & 0 \leq \underline{t_{5}} \leq 2 \\
80 \leq \underline{t_{2}} \leq 132 & 0 \leq \underline{t_{6}} \leq 28 \\
100 \leq \underline{t_{3}} \leq 100 & \underline{t_{5}}-\underline{t_{2}} \leq-80 \\
10 \leq \underline{t_{4}} \leq 20\end{cases}
\end{array}\right.
\end{aligned}
$$

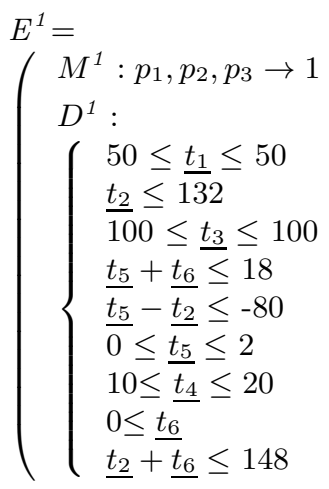

At this stage, the persistence of the inhibited transition $t_{6}$ induces polyhedral constraints that are non redundant, as represented in the system $D_{1}$. Therefore, the $D B M$ restriction induces an over-approximation of the exact class $E_{1}$. However, we notice that the implementation of our algorithm computes a tighter overapproximation. Concretely, the maximal residual time of the inhibited transition $t_{6}$ has decreased to 18 . This value is exactly approximated by our algorithm, while it stands at 28 by using $R O M E O$. If we look to the system $D_{1}$ given in its minimal form, we notice that this time distance is worked out from the constraints $0 \leq t_{5}$ and $\underline{t_{5}}+\underline{t_{6}} \leq 18$. This latter which is of a polyhedral form is removed, as well as the constraint $\underline{t_{2}}+\underline{t_{6}} \leq 148$, to determine the system $\widetilde{D_{1}^{\prime \prime}}$. The normalization is then applied to the set of $D B M$ constraints, whereas it should be done before 
removing the polyhedral constraints. By proceeding in this way, the $D B M$ overapproximation implemented in $R O M E O$ improves the complexity of the approach defined in [15], but at the expenses of the precision of the over-approximation.

Let us take a look at Figure 4 to explain why the residual time of the transition $t_{6}$ has decreased during its inhibition. This figure depicts the temporal scenario of the firing sequence $\left(t_{3}, t_{4}, t_{2}, t_{1}\right)$ leading to the class $\widetilde{E}_{1}$.

From the class enabling $t_{1}$ for the first time, the transition $t_{3}$ is fired without any delay. Subsequently, the newly enabled transition $t_{6}$ is inhibited, and we have $\widetilde{D}\left[\bullet, t_{6}\right]=28$. The firing of $t_{4}$ between $[10,20]$ yields a new class. There, $t_{6}$ becomes activated for the first time and its maximal residual time $\widetilde{D}\left[\bullet, t_{6}\right]=28$ stands as it was. The transition $t_{6}$ is then inhibited again after the firing of $t_{2}$ during $[0,22]$.

Then, to be able to fire the persistent transition $t_{1}$ it needs to let time progress with minimum $\operatorname{tmin}\left(t_{1}\right)=50$ from the start of the sequence. Within this intention, only the states (valuations), that fire $t_{2}$ during $[10,22]$ (while $t_{6}$ was activated), are satisfying the firing constraints of $t_{1}$. This restriction implies that the maximal residual time of $t_{6}$ decreases of 10 units (from 28 to 20), after the firing of $t_{1}$.

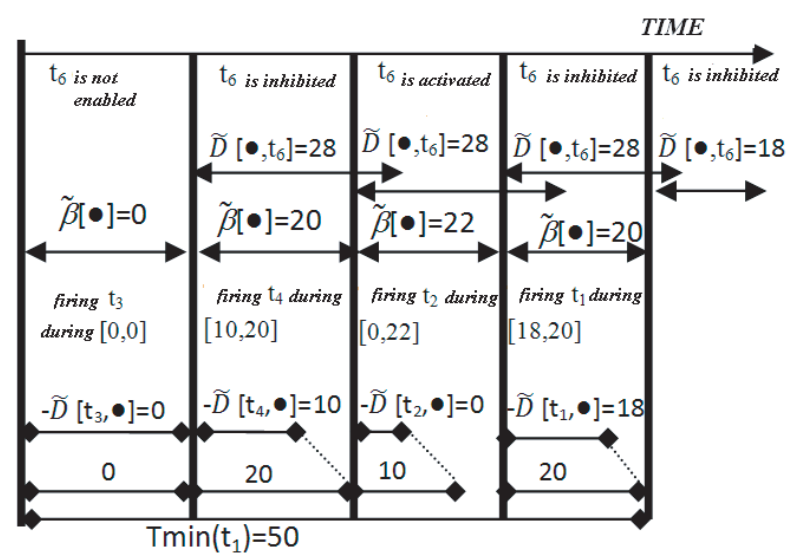

Figure 4: Temporal scenario of the firing sequence $\left(t_{3}, t_{4}, t_{2}, t_{1}\right)$.

The loss of precision reported in the $D B M$ over-approximation implemented in the tool $R O M E O$ can have an impact on the firability of yet persistent transitions. To illustrate this point, let us consider, for instance, the firing of the sequence $\left(t_{4}, t_{5}\right)$ from the classes $\widetilde{E^{1}}, \widetilde{E^{\prime \prime}} 1$ and $E^{1}$, leading respectively to the classes $\widetilde{E^{2}}, \widetilde{E^{\prime \prime 2}}$ and $E^{2}$. 


$\widetilde{E^{2}}=\left(\begin{array}{c|c|c|c|c|c|}M^{2}: p_{3} \rightarrow 1 \\ \hline \widetilde{D}^{2} & \bullet & t_{1} & t_{2} & t_{3} & t_{6} \\ \hline \bullet & 0 & 40 & 122 & 90 & 18 \\ \hline t_{1} & -28 & 0 & 82 & 50 & -10 \\ \hline t_{2} & -60 & -30 & 0 & 20 & -42 \\ \hline t_{3} & -78 & -50 & 32 & 0 & -60 \\ \hline t_{6} & 0 & 40 & 122 & 90 & 0 \\ \hline\end{array}\right.$

$$
\begin{aligned}
& \widetilde{E^{\prime \prime 2}}:= \\
& \left(\begin{array}{l}
M^{2}: p_{3} \rightarrow 1 \\
\widetilde{D^{\prime \prime 2}}: \\
\begin{cases}28 \leq \underline{t_{1}} \leq 40 & \underline{t_{1}}-\underline{t_{3}} \leq-50 \\
60 \leq \underline{t_{2}} \leq 122 & \underline{t_{2}}-\underline{t_{1}} \leq 82 \\
78 \leq \underline{t_{3}} \leq 90 & \underline{t_{2}}-\underline{t_{3}} \leq 32 \\
0 \leq \underline{t_{6}} \leq 28 & \underline{t_{3}}-\underline{t_{1}} \leq 50 \\
\underline{t_{1}}-\underline{t_{2}} \leq-30 & \underline{t_{3}}-\underline{t_{2}} \leq 20\end{cases}
\end{array}\right. \\
& E^{2}:=
\end{aligned}
$$

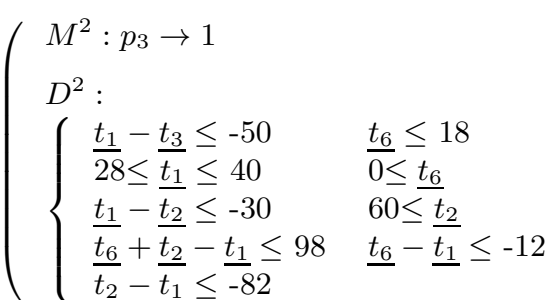

We notice that the transition $t_{1}$ is firable from the approximated class $\widetilde{E^{\prime \prime}}$, while it is not from the exact class $E^{2}$ (since $t_{6}-t_{1} \leq-12$ ), as well as when using our algorithm (since $\widetilde{D^{2}}\left[t_{1}, t_{6}\right]=-10<0$ ). This ensures that our construction yields a less coarse over-approximation than that implemented in ROMEO.

Comparatively to the implementation of the approach of [7] in $O R I S$ tool [16], the resulted $D B M$ systems are equivalent to the ones computed by our algorithm. Actually, the implemented algorithm in $O R I S$ proceeds by normalizing the precomputed systems before removing the non $D B M$ constraints. However, by proceeding in this way the durations needed to compute the graphs are higher as we will see in section 5 when reporting the simulation results. Moreover, although the graphs computed by $O R I S$ are different, they are bisimilar to those computed by our algorithm. Actually, a class is expressed in $O R I S$ as a tuple $(M, \widetilde{D}, N e w)$ where $N e w$ is a boolean function which indicates whether each transition enabled in the class is persistent or not. As the equivalence test implemented in $O R I S$ is based on the equality of all the three parameters ${ }^{7}$, this construction therefore yields coarser graphs.

In respect to the approach defined in [5], the latter proceeds by quantization, to over-approximate the exact polyhedra by another one. This approach is configurable, namely the precision of the approximation (grid), can be fixed before the graph computation. When using the thinnest grid, this approach succeeds to compute the exact graph in almost all cases but with the highest cost in terms of computation time and memory usage. However, as this technique still manipulates general polyhedra, it undergoes a higher computation complexity comparatively to $D B M$ over approximation.

To sum up, the exact computation as well as the K-grid approximation of the graph of the net of Figure 3 yield 320 classes. On the other hand, the $D B M$

\footnotetext{
${ }^{7}$ Only the equality of the parameters $M$ and $\widetilde{D}$ is needed to decide the equivalence, as evidenced in Definition 7.
} 
over-approximation computed by ROMEO contains 403 classes and that by using ORIS produces 429 classes. The implementation of the construction defined in this paper produces a more precise and compact graph of 394 classes.

We explore in the next section how to improve the construction of the $D B M$ over-approximation. This is achieved by reducing still more its size and the effort of its computation, but however while relaxing a little bit in the precision of the over-approximation.

\section{Efficient construction of a DBM over-approxi- mation}

We have proposed in the last section an efficient algorithm to compute the tightest $D B M$ over-approximation of an ITPN. We have shown that the firing interval $[-\widetilde{D}[t, \bullet], \widetilde{D}[\bullet, t]$ of a persistent inhibited transition may narrow along a firing sequence. However, in almost all cases this interval remains unchanged. Therefore, we propose in the sequel to relax a little bit the $D B M$ constraints so that to compute more compact graphs while reducing their computation effort. However, as this new abstraction is not as precise as that defined in Definition 6, it may therefore contain additional sequences. On the other hand, it makes it possible to compute in many cases, with lesser expenses, more compact graphs that are indeed bisimilar to $\widetilde{G R}$ and $G R$. Formally, this construction is defined as follows:

Definition 8. The contracted DBM over-approximation graph of an ITPN, denoted by $\overparen{G R C}$, is the tuple

$\left.\widetilde{(C E C}, \widetilde{E_{c}^{0}}, \hookrightarrow\right)$, such that :

- $\widetilde{C E C}$ is the set of DBM over-approximated classes reachable in $\widetilde{G R C}$;

- $\widetilde{E_{c}^{0}}=\left(M^{0}, \widetilde{D_{c}^{0}}\right) \in \widetilde{C E C}$ is the initial class such that $\widetilde{D_{c}^{0}}=\widetilde{D^{0}}=D^{0}$.

- $\hookrightarrow$ is a transition relation between DBM over-approximated classes defined on $\widetilde{C E C} \times T \times \widetilde{C E C}$, such that $\left(\left(M, \widetilde{D_{c}}\right), t_{f},\left(M^{\uparrow}, \widetilde{D_{c}^{\uparrow}}\right)\right) \in \rightsquigarrow$, iff :

$-\left(t_{f} \in T a(M)\right) \wedge\left(\widetilde{\beta_{c}}\left[t_{f}\right] \geq 0\right)$ such that: $\forall x \in T e(M) \cup\{\bullet\}, \widetilde{\beta_{c}}[x]=$ $\underset{\forall t \in \operatorname{Ta}(M)}{\operatorname{MIN}}\left\{\widetilde{D_{c}}[x, t]\right\}$.

- $\forall p \in P, M^{\uparrow}(p):=M(p)-B\left(p, t_{f}\right)+F\left(p, t_{f}\right)$.

- The coefficients of the DBM inequalities of the system $\widetilde{D}_{c}^{\uparrow}$ are computed from those of $\widetilde{D}_{c}$ by applying the following algorithm:

$\forall t \in T e\left(M^{\uparrow}\right)$

$$
\widetilde{D_{c}^{\uparrow}}[t, t]:=0 ; \quad \widetilde{D_{c}^{\uparrow}}[\bullet, \bullet]:=0 .
$$

If $t$ is persistent 


$$
\begin{aligned}
& \text { If } t \in T i(M) \quad \widetilde{D_{c}^{\uparrow}}[t, \bullet]:=\widetilde{D_{c}}[t, \bullet] ; \quad \widetilde{D_{c}^{\uparrow}}[\bullet, t]:=\widetilde{D_{c}}[\bullet, t] . \\
& \text { If } t \notin \operatorname{Ti}(M) \quad \widetilde{D_{c}^{\uparrow}}[\bullet, t]:=\widetilde{D_{c}}\left[t_{f}, t\right] ; \quad \widetilde{D_{c}^{\uparrow}}[t, \bullet]:=\widetilde{\beta_{c}}[t] \text {. } \\
& \text { If } t \text { is newly enabled. } \\
& \widetilde{D_{c}^{\uparrow}}[\bullet, t]:=\operatorname{tmax}(t) ; \quad \widetilde{D_{c}^{\uparrow}}[t, \bullet]:=-\operatorname{tmin}(t) . \\
& \forall\left(t_{1}, t_{2}\right) \in\left(T e\left(M^{\uparrow}\right)\right)^{2} \wedge\left(t_{1} \neq t_{2}\right) \\
& \text { If } t_{1} \text { or } t_{2} \text { are newly enabled. } \quad \widetilde{D_{c}^{\uparrow}}\left[t_{1}, t_{2}\right]:=\widetilde{D_{c}^{\uparrow}}\left[\bullet, t_{2}\right]+\widetilde{D_{c}^{\uparrow}}\left[t_{1}, \bullet\right] \text {. } \\
& \text { If } t_{1} \text { and } t_{2} \text { are persistent. } \\
& \text { If }\left(t_{1}, t_{2}\right) \notin(T i(M))^{2} \text { or }\left(t_{1}, t_{2}\right) \in(T i(M))^{2} \\
& \widetilde{D_{c}^{\uparrow}}\left[t_{1}, t_{2}\right]:=\operatorname{MIN}\left(\widetilde{D_{c}}\left[t_{1}, t_{2}\right], \quad \widetilde{D_{c}^{\uparrow}}\left[\bullet, t_{2}\right]+\widetilde{D_{c}^{\uparrow}}\left[t_{1}, \bullet\right]\right) . \\
& \text { If }\left(t_{1}, t_{2}\right) \notin(T i(M))^{2} \wedge\left(t_{1} \in T i(M)\right) \vee\left(t_{2} \in T i(M)\right) \\
& \widetilde{D_{c}^{\uparrow}}\left[t_{1}, t_{2}\right]:=\widetilde{D_{c}^{\uparrow}}\left[\bullet, t_{2}\right]+\widetilde{D_{c}^{\uparrow}}\left[t_{1}, \bullet\right] .
\end{aligned}
$$

Compared to the construction introduced in Definition 6, the last algorithm relaxes the constraints of persistent inhibited transitions. The firing interval of the latter is assumed unchanging, while the dwelling time in the class $\widetilde{E_{c}}$ is neglected when computing the firing distance between a persistent inhibited transition and a persistent activated transition. However, although this construction relaxes the constraints of each class of $\widetilde{G R}$, we need to prove formally that it computes in all cases an over-approximation of $\overline{G R}$, and hence of $G R$.

Theorem 2. The graph $\widetilde{G R C}=\left(\widetilde{C E},\left(M^{0}, \widetilde{D_{c}^{0}}\right), \hookrightarrow\right)$ is a DBM over-approximation of the graph $G R=\left(C E,\left(M^{0}, D^{0}\right), \longmapsto\right)$.

Proof. The proof is conducted in the same way as for the proof of Theorem.1. First of all, we notice that $\rceil D^{0}\lceil=\rceil \overrightarrow{D^{0}}\lceil=\rceil \widetilde{D^{0}}\lceil=\rceil \widetilde{D_{c}^{0}}\lceil$.

Then by performing the same manipulations on the system $\vec{D}$ we determine the system:

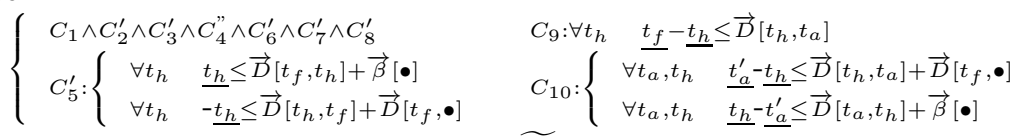

Then the constraints of the system $\widetilde{D_{c}^{\uparrow}}$ are obtained by cutting off the constraints of $C_{5}^{\prime}$ and $C_{10}$. Hence we obtain a system that is less precise than $\widetilde{D^{\uparrow}}$ and $\overrightarrow{D^{\uparrow}}$ and which defines an over-approximation of the system $\left.\left.\left.\overrightarrow{D^{\uparrow}}:\right\rceil \overrightarrow{D^{\uparrow}}\right\rceil=\right\rceil \widetilde{D^{\uparrow}}\lceil\subseteq$ \rceil$\widetilde{D_{c}^{\uparrow}}\lceil$.

We show in the sequel how the construction of the graph $\widetilde{G R C}$, as defined in Definition 8, can be improved still more by reducing as well as its size as the effort of its computation. For this effect, we explore hereafter an equivalence relation 
that is less restrictive than the equality which we prove to be a bisimulation. This means that the graphs resulted by using this bisimulation induces the same firing sequences as when using the equality.

We first give clues about the concepts used in this bisimulation, then we define it formally:

1. From Definition 8, we notice that the firing condition of a transition $t_{f}$ depends only on the sign of the coefficients $\widetilde{D}_{c}\left[t_{f}, t\right]$. Furthermore, the computation formulae of the system $\widetilde{D_{c}^{\uparrow}}$ from $\widetilde{D_{c}}$ does not use the elements $\widetilde{D_{c}}[\bullet, t]$ as well as $\widetilde{D}_{c}[t, \bullet]$ for $t \in T a(M)$. Therefore, as the latter coefficients are not involved in the firing tests, we need not to compare them when performing the class' equivalence test. This property makes it possible to gather classes that are not equal in the graph $\widetilde{G R C}$ but which enjoy indeed the same firing sequences.

2. Let us explore now other firing distances that are useless for the enumeration process. In order to investigate this point, first we need to introduce the following notation:

- A transition $t_{i}$ is said to be inhibiting $t_{j}$, if $\exists p \in P, \quad 0<I H\left(p, t_{i}\right) \leq$ $B\left(p, t_{j}\right)$. This means that if the transition $t_{j}$ is enabled for a given marking, then $t_{j}$ cannot be activated for this marking. We denote hereafter by Inhib the relation defined on $T^{2}$, such that $\left(t_{i}, t_{j}\right) \in I n h i b$, if $t_{j}$ is inhibiting $t_{i}$. Note that the relation Inhib is not symmetric; however if $\left(t_{i}, t_{j}\right)$ and $\left(t_{j}, t_{i}\right) \in I n h i b$, then this means that $t_{i}$ and $t_{j}$ are always inhibited when they are enabled together.

Let us consider two transitions that cannot be activated for a same marking (namely, $t^{\prime}$ is inhibiting $t$ ). When building the graph $\widetilde{G R C}$, it seems obvious that the time constraints of the transition $t$ have no impact on the firing of $t^{\prime}$, and conversely. Therefore, the distances $\widetilde{D_{c}}\left[t^{\prime}, t\right]$ and $\widetilde{D_{c}}\left[t, t^{\prime}\right]$ are useless since they are not required in the firing test since $t$ and $t^{\prime}$ cannot be activated together. Moreover, we need not even to compute these distances and to compare them when performing the equivalence test.

3. We explore now whether some distances can be left out when dealing with conflicting transitions. Before discussing this point, we need to introduce the following notations:

- We note $A T$ the set of transitions of $T$ that are not connected to any inhibitor arc: $t \in A T$, if $\nexists p \in P, I H(p, t) \neq 0$.

- Two transitions $t_{i}$ and $t_{j}$ are said to be twin, if $\forall p \in P, \quad I H\left(p, t_{i}\right)=$ $I H\left(p, t_{j}\right)$. This means that if two transitions are enabled for a given marking, then they are both either inhibited or activated for this marking. We denote hereafter by $T$ win the relation defined on $T^{2}$, such that 
$\left(t_{i}, t_{j}\right) \in T$ win, if $t_{i}$ and $t_{j}$ are twin. Note that $A T^{2} \subseteq T$ win and we have if $\left(t_{i}, t_{j}\right) \in$ Twin, then $\left(t_{j}, t_{i}\right) \in$ Twin.

In [6] the authors proposed to contract the state class graph of a $T P N$. They proved that the firing distances between two conflicting transitions are useless when their values stand positive. Furthermore, they show that it is not required to re-compute these distances in reachable classes as long as the conflicting transitions are not disabled ahead in the firing sequence.

In actual fact, within the context of $T P N$, if we have $\widetilde{D_{c}}\left[t, t^{\prime}\right] \geq 0$, then the transition $t^{\prime}$ has no impact on the firing of $t$ as long as both remain persistent. However, if $t$ is fired, then $t^{\prime}$ is disabled afterwards.

For an $I T P N$, the enforcement of this property may be inconsistent when dealing with conflicting transitions that are likely to be inhibited ahead in the firing sequence. For such transitions, the problem occurs, for instance, when we have two conflicting transitions $t^{\prime}$ and $t$ activated for two different classes $\widetilde{E_{c}}$ and $\widetilde{E_{c}^{\prime}}$ such that $\widetilde{D_{c}}\left[t, t^{\prime}\right] \neq \widetilde{D_{c}}\left[t, t^{\prime}\right] \geq 0$. If we consider these two classes as equivalent, they might not be bisimilar indeed. To be concrete, let us assume that a sequence is fired from both $\widetilde{E_{c}}$ and $\widetilde{E_{c}^{\prime}}$ during which $t$ is inhibited. Then $t$ becomes activated in the reachable classes $\widetilde{E_{c}^{\uparrow}}$ and $\widetilde{E_{c}^{\uparrow \prime}}$. At this stage, the distance $\widetilde{D_{c}^{\uparrow}}\left[t, t^{\prime}\right]$ may change to negative in $\widetilde{E_{c}^{\uparrow}}$ but there is no guarantee that the distance $\widetilde{D_{c}^{\uparrow \prime}}\left[t, t^{\prime}\right]$ may change too in $\widetilde{E_{c}^{\uparrow \prime}}$. Hence, $t$ may not be firable from $\widetilde{E_{c}^{\uparrow}}$, while it might be from $\widetilde{E_{c}^{\uparrow \prime}}$. Therefore, at first glance, we should restrict the application of this property only to conflicting transitions of $A T$; those which are not connected to any inhibitor arc. However, we show that under some assumptions the application of this property can be also extended to inhibited transitions. Actually, to validate this contraction as a bisimulation, we need to ensure that both transitions have been inhibited during the same periods of time. To guarantee that the last condition holds, we need only to assume that the conflicting transitions $t^{\prime}$ and $t$ are twin .

To illustrate this bisimulation, let us consider the ITPN of Figure 5. $a$ where we have $I n h i b=\left\{\left(t_{4}, t_{3}\right),\left(t_{5}, t_{3}\right)\right\}$. According to the previous discussion, the distances $\widetilde{D_{c}}\left[t_{4}, t_{3}\right], \widetilde{D_{c}}\left[t_{3}, t_{4}\right], \widetilde{D_{c}}\left[t_{3}, t_{5}\right]$ and $\widetilde{D_{c}}\left[t_{5}, t_{3}\right]$ should be left out during the computation of any class of the graph as well as when performing the equivalence test. Furthermore, we have $A T=\left\{t_{1}, t_{2}, t_{3}, t_{6}\right\}$ and $T$ win $=A T^{2} \cup\left\{\left(t_{4}, t_{5}\right),\left(t_{5}, t_{4}\right)\right\}$. However, among elements of Twin, only transitions $t_{1}$ and $t_{2}$, on a hand, and $t_{4}$ and $t_{5}$, on the other hand, are in conflict for the initial marking. Therefore, since the distances $\widetilde{D_{c}^{0}}\left[t_{1}, t_{2}\right], \widetilde{D_{c}^{0}}\left[t_{2}, t_{1}\right], \widetilde{D_{c}^{0}}\left[t_{4}, t_{5}\right]$ and $\widetilde{D_{c}^{0}}\left[t_{5}, t_{4}\right]$ are positive, we need not to re-compute their values as long as the related transitions remain persistent. Furthermore, as the firing of $t_{1}\left(\mathrm{resp}, t_{4}\right)$, disables $t_{2}\left(\mathrm{resp}, t_{5}\right)$, and conversely, we need not too to consider these distances for the equivalence test.

The exact construction $G R$, the tightest $D B M$ over-approximation $\widetilde{G R}$ and the abstraction $\widetilde{G R C}$ produce all the same graph shown in Figure 5.b. However, the 


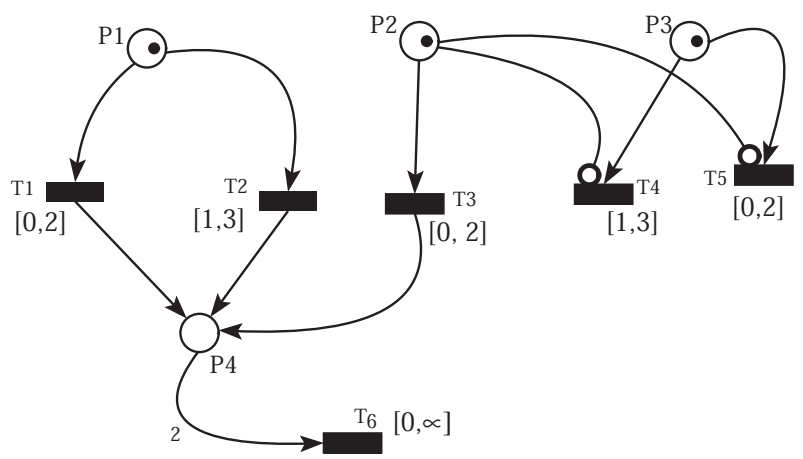

(a)

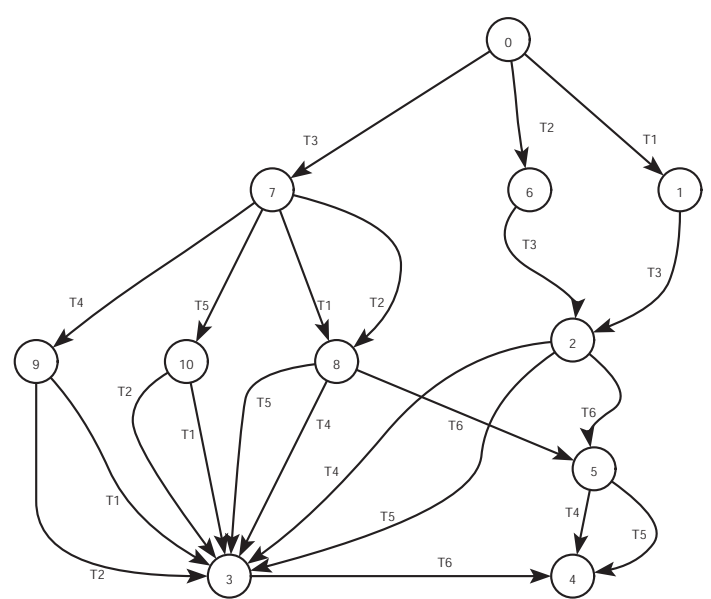

(b)

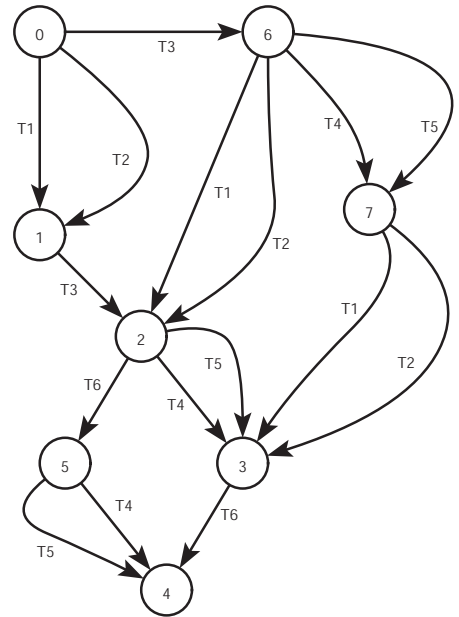

(c)

Figure 5: An ITPN model with twin and inhibiting transitions, with its reachability graphs.

application of the last properties makes it possible to contract further the graph $\widetilde{G R C}$, as depicted in Figure 5.c. Although it is smaller, the resulted graph still remains bisimilar to the former, as it allows gathering classes that derive from the same firing sequences ${ }^{8}$. For instance, firing $t_{1}$ (resp, $t_{2}$ ), in $\widetilde{G R C}$ from the initial class $\widetilde{E_{c}^{0}}$ leads to the class ${ }^{9} \widetilde{E_{c}^{1}}\left(\mathrm{resp}, \widetilde{E_{c}^{6}}\right)$. In actual fact, the distances $\widetilde{D_{c}}\left[\bullet, t_{3}\right], \widetilde{D_{c}}\left[t_{4}, t_{3}\right]$ and $\widetilde{D_{c}}\left[t_{5}, t_{3}\right]$ which impede the equality to hold, are useless since $t_{3}$ is inhibiting $t_{4}$ and $t_{5}$. Furthermore, the classes $\widetilde{E_{c}^{2}}$ and $\widetilde{E_{c}^{8}}$ are equivalent since the value of the minimal residual time of $t_{4}$ can be left out. Finally, the classes $\widetilde{E_{c}^{10}}$ and $\widetilde{E_{c}^{9}}$ can be gathered since the positive distances $\widetilde{D_{c}}\left[t_{1}, t_{2}\right]$ can be ignored;

\footnotetext{
${ }^{8}$ These classes are not equal, but they are bisimilar indeed.

${ }^{9}$ The class $\widetilde{E_{c}^{i}}$ corresponds to the node numbered $(i)$ in the graph.
} 
$t_{1}$ and $t_{2}$ are two twin conflicting transitions. Hence we obtain a more compact graph of 8 nodes and 16 edges, whereas the other constructions produce a graph of 11 classes and 22 edges.

$\widetilde{E_{c}^{0}}=\left(\begin{array}{|c|c|c|c|c|c|c|}M^{0}: p_{1}, p_{2}, p_{3} \rightarrow 1 \\ \hline D_{c}^{0} & \bullet & t_{1} & t_{2} & t_{3} & t_{4} & t_{5} \\ \hline \bullet & 0 & 2 & 3 & 2 & 3 & 2 \\ \hline t_{1} & 0 & 0 & 3 & 2 & 3 & 2 \\ \hline t_{2} & -1 & 1 & 0 & 1 & 2 & 1 \\ \hline t_{3} & 0 & 2 & 3 & 0 & 3 & 2 \\ \hline t_{4} & -1 & 2 & 2 & 1 & 0 & 1 \\ \hline t_{5} & 0 & 2 & 3 & 2 & 3 & 0 \\ \hline\end{array}\right.$

$\widetilde{E_{c}^{1}}=\left(\begin{array}{c|c|c|c|c|}M^{1}: p_{2}, p_{3}, p_{4} \rightarrow 1 \\ \hline \widetilde{D}_{c}^{1} & \bullet & t_{3} & t_{4} & t_{5} \\ \hline \bullet & 0 & 2 & 3 & 2 \\ \hline t_{3} & 0 & 0 & 3 & 0 \\ \hline t_{4} & 1 & 1 & 0 & 1 \\ \hline t_{5} & 0 & 2 & 3 & 0 \\ \hline\end{array}\right.$
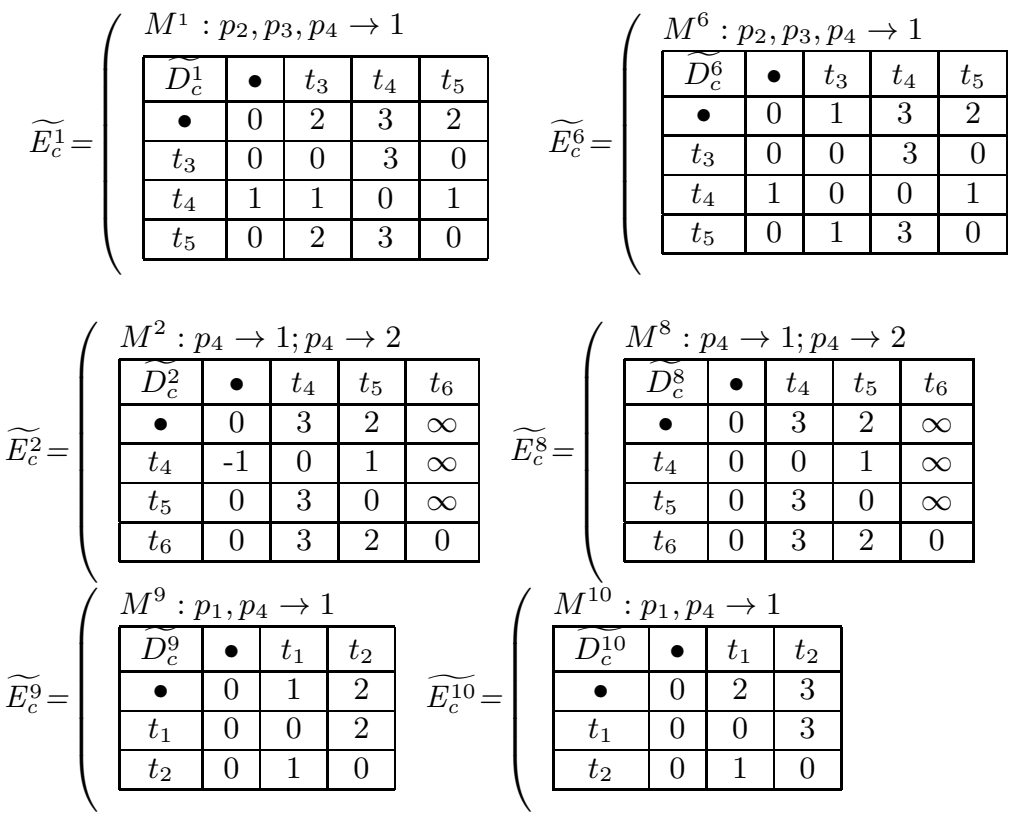

$\widetilde{E_{c}^{10}}=\left(\begin{array}{c|c|c|c|}M^{10}: p_{1}, p_{4} \rightarrow 1 \\ \hline \widetilde{D_{c}^{10}} & \bullet & t_{1} & t_{2} \\ \hline \bullet & 0 & 2 & 3 \\ \hline t_{1} & 0 & 0 & 3 \\ \hline t_{2} & 0 & 1 & 0 \\ \hline\end{array}\right.$

More formally, we introduce this contraction as an equivalence relation, defined as given next:

Definition 9. Let $\simeq$ be a relation over state classes of the graph $\widetilde{G R C}$, defined by: $\left(\left(M, \widetilde{D_{c}}\right),\left(M^{\prime}, \widetilde{D_{c}^{\prime}}\right)\right) \in \simeq$ iff:

(i) $M=M^{\prime}$

(ii) $\forall t \in T i(M) \quad \widetilde{D_{c}}[\bullet, t]=\widetilde{D_{c}^{\prime}}[\bullet, t], \quad \widetilde{D_{c}}[t, \bullet]=\widetilde{D_{c}^{\prime}}[t, \bullet]$

(iii) $\forall\left(t, t^{\prime}\right) \in$ Twin $\cap \operatorname{Conf}(M)$

$$
\left\{\begin{array}{l}
s g\left(\widetilde{D_{c}}\left[t, t^{\prime}\right]\right)=s g\left(\widetilde{D_{c}^{\prime}}\left[t, t^{\prime}\right]\right) \\
\widetilde{D_{c}}\left[t, t^{\prime}\right]=\widetilde{D_{c}^{\prime}}\left[t, t^{\prime}\right]
\end{array} \quad \text { If } s g\left(\widetilde{D_{c}}\left[t, t^{\prime}\right]\right)=<_{0}\right.
$$

(iv) $\forall\left(t, t^{\prime}\right) \in T e(M)^{2}-($ Twin $\cap$ Conf $(M))$ such that $\left(t^{\prime}, t\right),\left(t, t^{\prime}\right) \notin$ Inhib,

$$
\widetilde{D_{c}}\left[t, t^{\prime}\right]=\widetilde{D_{c}^{\prime}}\left[t, t^{\prime}\right] .
$$


where $s g(v)$ is a function which gives the sign of the value $v, s g: \mathbb{Q} \cup\{\infty\} \longrightarrow$ $\left\{\geq_{0},<_{0}\right\}$ such that $\geq_{0}$ (resp, $\left.<_{0}\right)$, denotes "positive or null" (resp, strictly negative).

In concrete terms, two classes $\left(M, \widetilde{D_{c}}\right)$ and $\left(M^{\prime}, \widetilde{D_{c}^{\prime}}\right)$ are in the relation $\simeq$, iff: ( $i$ ) they enjoy the same marking; $(i i)$ the maximum and the minimum residual times of any inhibited transition must be equal in both classes; ( $i i i$ ) for any pair of conflicting twin enabled transitions, the firing distances involving both transitions in both classes hold the same sign, and these distances must be equal in both classes only when they are negative; $(i v)$ For all other pairs of enabled transitions that are not in the relation Inhib, the firing distance involving both transitions must be equal. Let us prove now that the relation $\simeq$ is a bisimulation over the classes of the graph $\widetilde{G R C}$.

Theorem 3. The relation $\simeq$ is a bisimulation over the graph $\widetilde{G R C}$.

Proof. We should prove that if $\left(\widetilde{E_{c}}, \widetilde{E_{c}^{\prime}}\right)$ satisfies the hypotheses of Definition 9, then we have:

1 : If an activated transition $t_{f}$ can fire from $\widetilde{E}_{c}$, then $t_{f}$ can fire from $\widetilde{E_{c}^{\prime}}$ too. 2: If ${\widetilde{E_{c}}}_{c}^{t_{f}} \widetilde{E_{c}^{\uparrow}} \wedge \widetilde{E_{c}^{\prime}} \stackrel{t_{f}}{\hookrightarrow} \widetilde{E_{c}^{\prime \uparrow}}$, then $\left.\widetilde{\left(E_{c}^{\uparrow}\right.}, \widetilde{E_{c}^{\prime \uparrow}}\right) \in \simeq ; \widetilde{E_{c}^{\uparrow}}$ and $\widetilde{E_{c}^{\prime \uparrow}}$ satisfy Definition

9 .

1. Let us assume that the transition $t_{f}$ is firable from $\widetilde{E_{c}}=\left(M, \widetilde{D_{c}}\right)$. As $\widetilde{E_{c}}$ and $\widetilde{E_{c}^{\prime}}$ are in the relation $\simeq$, then the hypotheses of Definition 9 are satisfied. Basing on the firing condition, we need to prove that $(\mathrm{A} 1)$ : if $\widetilde{\beta_{c}}\left[t_{f}\right] \geq 0$, then $\widetilde{\beta_{c}^{\prime}}\left[t_{f}\right] \geq 0$, namely that $\underset{\forall t^{\prime} \in \operatorname{Mi}(M)}{\operatorname{IN}}\left\{\widetilde{D_{c}^{\prime}}\left[t_{f}, t^{\prime}\right]\right\} \geq 0$. As $t_{f}$ and $t^{\prime}$ are both activated, then $\left(t_{f}, t^{\prime}\right),\left(t^{\prime}, t_{f}\right) \notin I n h i b$. Hence, from hypotheses $(i i i)$ and $(i v)$ of Definition 9 we determine the property $(\mathrm{A} 1) ; t_{f}$ is firable from $\widetilde{E^{\prime}}$.

2. We have to prove that the hypotheses of Definition 9 are satisfied for $\left(\widetilde{E_{c}^{\uparrow}, \widetilde{E_{c}^{\prime \uparrow}}}\right)$

(a) It is obvious that as $M=M^{\prime}$, we have $M^{\uparrow}=M^{\uparrow \uparrow}$.

(b) Let us prove that $\left.\forall t \in T i\left(M^{\uparrow}\right), \widetilde{D_{c}}[\bullet, t]=\widetilde{{D_{c}^{\prime \uparrow}}^{\top}} \bullet \bullet, t\right]$. Let us replace $\widetilde{D_{c}^{\uparrow}}[\bullet, t]$ with its computation formula according to the status of $t$, as given in Definition 8

if $t \in N e w\left(M^{\uparrow}\right)$, then we have $: \widetilde{D_{c}^{\uparrow}}[\bullet, t]=\widetilde{{D_{c}^{\prime \uparrow}}^{\prime}}[\bullet, t]=\operatorname{tmax}(t)$.

if $t \notin N e w\left(M^{\uparrow}\right)$, then we should consider whether $t$ is inhibited for $M$ or not.

- If $t \notin T i(M)$, then we need to prove that $\widetilde{D_{c}}\left[t_{f}, t\right]=\widetilde{D_{c}^{\prime}}\left[t_{f}, t\right]$. This last property holds since $\left(t_{f}, t\right) \notin$ Twin $\cap \operatorname{Conf}(M)$, otherwise $t$ should be disabled after firing $t_{f}$. Furthermore, $\left(t_{f}, t\right) \notin I n h i b$ otherwise $t_{f}$ should be inhibited for $M$. Also $\left(t, t_{f}\right) \notin I n h i b$, otherwise $t$ should be inhibited for $M$. 
- If $t \in T i(M)$, then the proof is obvious from the hypothesis $(i i)$.

Notice that likewise we can also prove that $\forall t \in T a\left(M^{\uparrow}\right), \widetilde{D_{c}}[\bullet, t]=$ $\widetilde{D_{c}^{\prime \uparrow}}[\bullet, t]$.

(c) We should prove that $\forall t \in T i\left(M^{\uparrow}\right), \widetilde{D_{c}^{\uparrow}}[t, \bullet] \widetilde{D_{c}^{\prime \uparrow}}[t, \bullet]$. Let us replace $\widetilde{D_{c}^{\uparrow}}[t, \bullet]$ with the suitable computation formula according to the status of the transition $t$.

if $t \in N e w\left(M^{\uparrow}\right)$, then we have $\widetilde{D_{c}^{\uparrow}}[t, \bullet]=\widetilde{D_{c}^{\uparrow}}[t, \bullet]=-\operatorname{tmin}(t)$.

if $t \notin N e w\left(M^{\uparrow}\right)$, then we should consider whether $t$ is inhibited for $M$ or not.

- If $t \notin T i(M)$, then we need to prove that $\widetilde{\beta_{c}^{\uparrow}}[t]=\widetilde{\beta_{c}^{\uparrow}}[t]$, namely that $\underset{\forall t^{\prime} \in \operatorname{Ti}(M)}{\operatorname{IN}}\left\{\widetilde{D_{c}}\left[t, t^{\prime}\right]\right\}=\underset{\forall t^{\prime} \in \operatorname{Ti}(M)}{\operatorname{MIN}}\left\{\widetilde{D_{c}^{\prime}}\left[t, t^{\prime}\right]\right\}$.

If $\left(t, t^{\prime}\right) \notin($ Twin $\cap \operatorname{Conf}(M)) \cup \operatorname{Inhib}$, then we have $\widetilde{D_{c}}\left[t, t^{\prime}\right]=$ $\widetilde{D_{c}^{\prime}}\left[t, t^{\prime}\right]$. However, $\left(t^{\prime}, t\right) \notin \operatorname{Inhib}\left(\operatorname{resp},\left(t, t^{\prime}\right) \notin\right.$ Inhib), otherwise $t^{\prime}$ must be inhibited (resp, $t$ must be inhibited), for $M$.

If $\left(t, t^{\prime}\right) \in T$ win $\cap \operatorname{Con} f(M)$, then we have $s g\left(\widetilde{D}\left[t, t^{\prime}\right]\right)=s g\left(\widetilde{D^{\prime}}\left[t, t^{\prime}\right]\right)$ and yet more $\widetilde{D_{c}}\left[t, t^{\prime}\right]=\widetilde{D_{c}^{\prime}}\left[t, t^{\prime}\right]$ when $s g\left(\widetilde{D}\left[t, t^{\prime}\right]\right)=<_{0}$. As $t \in$ $T a(M)$ and $\widetilde{D_{c}}[t, t]=0, \operatorname{then}_{\forall t^{\prime} \in \operatorname{Ma}(M)}\left\{\widetilde{D_{c}}\left[t, t^{\prime}\right]\right\} \leq 0$. Therefore the value of $\widetilde{D_{c}}\left[t, t^{\prime}\right]$ has no effect on the calculation of the minimum when $s g\left(\widetilde{D_{c}}\left[t, t^{\prime}\right]\right)=\geq_{0}$; hence the equality holds.

- If $t \in T i(M)$, then the proof is stemmed from the hypothesis $(i i)$.

Notice that likewise we can also prove that $\forall t \in T a\left(M^{\uparrow}\right), \widetilde{D_{c}^{\uparrow}}[t, \bullet] \widetilde{D_{c}^{\prime \uparrow}}[t, \bullet]$.

(d) We have to prove that $(\mathrm{A} 2): \forall\left(t, t^{\prime}\right) \in \operatorname{Twin} \cap \operatorname{Conf}\left(M^{\uparrow}\right)$

$$
\left\{\begin{array}{l}
s g\left(\widetilde{D_{c}^{\uparrow}}\left[t, t^{\prime}\right]\right)=\operatorname{sg}\left(\widetilde{D_{c}^{\uparrow}}\left[t, t^{\prime}\right]\right) \\
\widetilde{D_{c}^{\uparrow}}\left[t, t^{\prime}\right]=\widetilde{D_{c}^{\prime \uparrow}}\left[t, t^{\prime}\right]
\end{array} \quad \text { If } s g\left(\widetilde{D_{c}^{\uparrow}}\left[t, t^{\prime}\right]\right)=<_{0}\right.
$$

As we deal with safe nets, we can easily show that if two persistent transitions are in conflict for $M$, then they remain in conflict for $M^{\uparrow}$. Hence, $\operatorname{Conf}\left(M^{\uparrow}\right)$ consists of all the pairs of transitions $\left(t, t^{\prime}\right) \in \operatorname{Conf}(M)$ that are persistent in $M^{\uparrow}$ to which we add the pairs of conflicting transitions for $M^{\uparrow}$ where at least one transition is newly enabled. First of all, it is obvious that if $t \in N e w\left(M^{\uparrow}\right)$ or $t^{\prime} \in N e w\left(M^{\uparrow}\right)$, then $\left(t, t^{\prime}\right)$ satisfies the hypothesis (A2).

Let us discuss the case where two twin conflicting transitions are persistent, $\left(t, t^{\prime}\right) \notin\left(N e w\left(M^{\uparrow}\right)\right)^{2}$. Therefore, according to Definition 8, we have:

$\widetilde{D_{c}^{\uparrow}}\left[t, t^{\prime}\right]=\operatorname{MIN}\left(\widetilde{D_{c}}\left[t, t^{\prime}\right], \widetilde{D_{c}^{\uparrow}}[t, \bullet] \widetilde{D_{c}^{\uparrow}}\left[\bullet, t^{\prime}\right]\right)$.

As it is assumed that $s g\left(\widetilde{D_{c}}\left[t, t^{\prime}\right]\right)=s g\left(\widetilde{D_{c}^{\prime}}\left[t, t^{\prime}\right]\right)$, and we have already 
proved through $(b),(c)$ that $(\mathrm{A} 3): \forall t \in T e(M), \widetilde{D_{c}^{\uparrow}}[\bullet, t]=\widetilde{D_{c}^{\prime \uparrow}}[\bullet, t]$ and $\widetilde{D_{c}^{\uparrow}}[t, \bullet]=\widetilde{D_{c}^{\prime \uparrow}}[t, \bullet]$, we can easily determine that $s g\left(\widetilde{D_{c}^{\uparrow}}\left[t, t^{\prime}\right]\right)=$ $\operatorname{sg}\left(\widetilde{D_{c}^{\prime \uparrow}}\left[t, t^{\prime}\right]\right)$.

Furthermore, if $s g\left(\widetilde{D_{c}^{\uparrow}}\left[t, t^{\prime}\right]\right)=s g\left(\widetilde{D_{c}^{\prime \uparrow}}\left[t, t^{\prime}\right]\right)=<_{0}$, then we should prove that $\widetilde{D_{c}^{\uparrow}}\left[t, t^{\prime}\right]=\widetilde{D_{c}^{\prime \uparrow}}\left[t, t^{\prime}\right]$; two cases can be seen:

- $\widetilde{D_{c}^{\uparrow}}\left[t, t^{\prime}\right]=\widetilde{D_{c}}\left[t, t^{\prime}\right]:$ as $\widetilde{D_{c}}\left[t, t^{\prime}\right]=\widetilde{D^{\prime}}\left[t, t^{\prime}\right]$ when $s g\left(\widetilde{D_{c}}\left[t, t^{\prime}\right]\right)=<_{0}$, we guarantee that $\widetilde{D_{c}^{\uparrow}}\left[t, t^{\prime}\right]=\widetilde{D_{c}^{\uparrow}}\left[t, t^{\prime}\right]$.

- $\widetilde{D_{c}^{\uparrow}}\left[t, t^{\prime}\right]=\widetilde{D_{c}^{\uparrow}}[t, \bullet]+\widetilde{D_{c}^{\uparrow}}\left[\bullet, t^{\prime}\right]$ : the property (A3) guarantees that $\widetilde{D_{c}^{\uparrow}}\left[t, t^{\prime}\right]=\widetilde{D_{c}^{\prime \uparrow}}\left[t, t^{\prime}\right]$.

(e) We have to prove that (A4): $\forall\left(t, t^{\prime}\right) \in T e\left(M^{\uparrow}\right)^{2}-\left(\left(\right.\right.$ Twin $\left.\cap \operatorname{Conf}\left(M^{\uparrow}\right)\right)$,

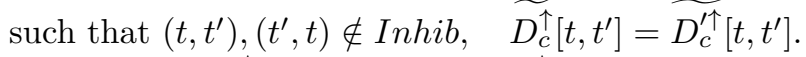

If $t \in N e w\left(M^{\uparrow}\right)$ or $t^{\prime} \in N e w\left(M^{\uparrow}\right)$, then by using property (A3) we prove that,

$\widetilde{D_{c}^{\uparrow}}\left[t, t^{\prime}\right]=\widetilde{{D_{c}^{\uparrow}}_{c}^{1}}\left[t, t^{\prime}\right]=\widetilde{D_{c}^{\uparrow}}[t, \bullet] \widetilde{D_{c}^{\uparrow}}\left[\bullet, t^{\prime}\right]=\widetilde{{D_{c}^{\uparrow}}^{\uparrow}}[t, \bullet] \widetilde{{D_{c}^{\uparrow}}_{c}^{\uparrow}}\left[\bullet, t^{\prime}\right]$.

Let us discuss the case where both transitions are persistent; we have either

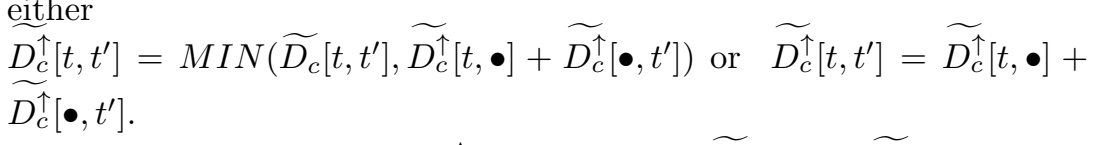
As $\left(t, t^{\prime}\right) \notin\left(\right.$ Twin $\cap \operatorname{Conf}\left(M^{\uparrow}\right) \cup \operatorname{Inhib}$, then $\widetilde{D_{c}}\left[t, t^{\prime}\right]=\widetilde{D_{c}^{\prime}}\left[t, t^{\prime}\right]$; hence the property (A4) holds.

\subsection{Discussion}

By avoiding, on one hand, to compute some distances when working out each reachable class, and on the other hand, to compare them during the equivalence test, we succeed in reducing the computation effort of the approximated graph $\widetilde{G R C}$. This construction achieves, in general, to significantly reduce the size of the graphs, but however loses a bit in the precision of the approximation. In fact, the relaxation of some $D B M$ constraints in the abstraction $\widehat{G R C}$ allows to gather many classes as equivalent even when they are not equal. On the other hand, these classes are not considered as equivalent in the other constructions $G R$ and $\widetilde{G R}$ although they often stand bisimilar.

For example, let us go back to the net of Figure.3 and let us consider again the sequence leading to the classes $\widetilde{E^{2}}, \widetilde{E^{\prime \prime}}$ and $E^{2}$ (see page 20). Through the same sequence we reach the class $\widetilde{E_{c}^{2}}$ in the graph $\widetilde{G R C}$. As it is the case for the $D B M$ over-approximation defined in [15], the class $\widetilde{E_{c}^{2}}$ allows the firing of transition $t_{1}$. On the other hand, the exact construction [13], the K-grid approximation [5], the 
algorithm introduced in Definition.6 as well as the approach defined in [7] do not. Actually, the firing of the transition $t_{1}$ is due to the relaxation of the constraints of the transition $t_{6}$ in $\widetilde{E_{c}^{2}}$. However, despite that the abstraction $\widetilde{G R C}$ does not build the tightest $D B M$ over-approximation, it succeeds in some cases to compute much compact graphs than other approaches. For instance, the resulted graph obtained for the net of Figure. 3 contains only 309 classes.

$\widetilde{E_{c}^{2}}=\left(\begin{array}{c|c|c|c|c|c|}M^{2}: p_{3} \rightarrow 1 \\ \hline \widetilde{D}_{c}^{2} & \bullet & t_{1} & t_{2} & t_{3} & t_{6} \\ \hline \bullet & 0 & 40 & 122 & 90 & 28 \\ \hline t_{1} & -28 & 0 & 82 & 50 & 0 \\ \hline t_{2} & -58 & -30 & 0 & 20 & -30 \\ \hline t_{3} & -78 & -50 & 32 & 0 & -50 \\ \hline t_{6} & 0 & 40 & 122 & 90 & 0 \\ \hline\end{array}\right.$

To illustrate this fact, let us consider the firing of the transition $t_{6}$ from the previous classes $\widetilde{E^{2}}, \widetilde{E_{c}^{2}}, \widetilde{E^{\prime \prime} 2}$ and $E^{2}$ to reach respectively the classes $\widetilde{E}^{3}, \widetilde{E_{c}^{3}}, \widetilde{E^{\prime \prime} 3}$ and $E^{3}$

$\widetilde{E^{3}}=\left(\begin{array}{c|c|c|c|c|}M^{3}: \rightarrow 0 \\ \hline D^{3} & \bullet & t_{1} & t_{2} & t_{3} \\ \hline \bullet & 0 & 40 & 122 & 90 \\ \hline t_{1} & -10 & 0 & 82 & 50 \\ \hline t_{2} & -42 & -30 & 0 & 20 \\ \hline t_{3} & -60 & -50 & 32 & 0 \\ \hline\end{array}\right.$

$\widetilde{E_{c}^{3}}=\left(\begin{array}{c|c|c|c|c|}M^{3}: \rightarrow 0 \\ \hline \widetilde{D_{c}^{3}} & \bullet & t_{1} & t_{2} & t_{3} \\ \hline \bullet & 0 & 40 & 122 & 90 \\ \hline t_{1} & -28 & 0 & 82 & 50 \\ \hline t_{2} & -30 & -30 & 0 & 20 \\ \hline t_{3} & -50 & -50 & 32 & 0 \\ \hline\end{array}\right.$

$$
\begin{aligned}
& E^{3}:= \\
& \left(\begin{array}{l}
M^{3}: \rightarrow 0 \\
D^{3}: \\
\left\{\begin{array}{c}
12 \leq \underline{t_{1}} \leq 40 \\
t_{1}-\underline{t_{2}} \leq-30
\end{array} \quad \underline{t_{1}}-\underline{t_{2}}-\underline{t_{1}} \leq-50\right.
\end{array}\right.
\end{aligned}
$$$$
\widetilde{E^{\prime \prime} 3}:=
$$$$
\left\{\begin{array}{l}
\widehat{M^{3}}: \rightarrow 0 \\
\widehat{D^{\prime \prime} 3}: \\
\left\{\begin{array}{l}
0 \leq t_{1} \leq 40 \\
32 \leq \underline{t_{2}} \leq 122 \\
50 \leq \underline{t_{3}} \leq 90
\end{array} \quad \underline{t_{1}}-\underline{t_{1}}-\underline{t_{3}} \leq-30\right. \\
\end{array}\right.
$$

As we can see, all the resulted classes contain only $D B M$ constraints. However the ones computed by the exact approach and the K-grid based approximation are the tighter ones (see the class $E^{3}$ ). On the other side, the $D B M$ overapproximations introduced in Definition.6 and in [7] produce the same class $\widetilde{E^{3}}$, whereas the one defined in [15] as well as the construction $\widetilde{G R C}$ compute less precise $D B M$ systems (see $\widetilde{E^{\prime \prime} 3}$ and $\widetilde{E_{c}^{3}}$ ). Although all these classes are unequal, they still derive the same firing sequences. Furthermore, the classes $\widetilde{E^{3}}, \widetilde{E^{\prime \prime}}$ and $E^{3}$ stand unique and are not equal to any other reachable class in their related graphs. However, the use of the equivalence $\simeq$ rather than the equality in the construction of $\widetilde{G R C}$ makes it possible to gather in a same node four other classes that stand bisimilar to $\widetilde{E_{c}^{3}}$ in the graph $\widetilde{G R C}$. Actually, according to Definition 9, the constraints $0 \leq \underline{t_{1}} \leq 40,32 \leq \underline{t_{2}} \leq 122$ and $50 \leq \underline{t_{3}} \leq 90$ in the class $\widetilde{E_{c}^{3}}$ are not needed 
to carry out the equivalence test. Therefore, despite the loss of precision in the over-approximation (which may induce additional sequences in the graph $\widetilde{G R C}$ ), this construction still computes a much compact graph than all other approaches.

Consequently, the abstraction over the classes of the graph $\widetilde{G R C}$ is the quotient graph of $\widetilde{G R C}$ w.r.t the relation $\simeq$. It preserves, markings and both firing sequences while it is, in general, smaller. The $\widetilde{G R C}$ may be more appropriate than $\widetilde{G R}$ to check over linear properties of the model, especially when the number of additional sequences that have been added due to constraint relaxation is limited. However, when the graph $\widetilde{G R C}$ provides a too coarse over-approximation, it may yield a larger graph than $\widetilde{G R}$; the additional sequences are too numerous to be wrapped by the contraction. Indeed, the construction of $\widetilde{G R C}$ is more convenient to build when many inhibiting and conflicting transitions are reported in the net, otherwise the construction of $\overline{G R}$ should be considered. In other respects, it should be noticed that all the sequences firable in $G R$ are preserved in $\widetilde{G R}$ and hence in the $\widetilde{G R C}$.

\section{Experimental results}

We have implemented the algorithm using $C++$ builder language on a Windows workstation. The graph construction is based on breadth-first graph generation search strategy. The experiments have been performed on a Pentium $V$ with a processor speed of 2,7 GHZ and 1,9 GB of memory capacity. The different tests have been carried out by using different tools: TINA tool[18], ROMEO tool[17], ORIS tool[16] and our tool named ITPNT.

The performances of the experiments are assessed by considering three parameters, the number of classes, the number of edges, and finally in terms of computation times. It is noteworthy that ROMEO and ORIS tool do not bring out some parameters; we denote that by the notation NA (Not Available). Also, we denote by NF (Not Finished) the tests that had led to memory overflows or to a big time computation; more than 5 minutes.

Through the first experiments we have checked whether the TPN graph con-

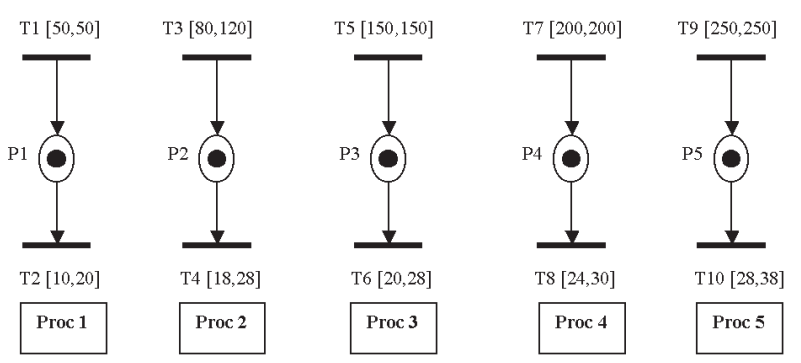

Figure 6: TPN used in the experiments. 
Table 2: Results of experiments performed on $T P N$.

\begin{tabular}{|c|c|c|c|c|c|}
\hline Examples & Tools & TINA & $R O M E O$ & $O R I S$ & $I T P N T$ \\
\hline \multirow{3}{*}{ Proc 1} & Classes & 2 & 2 & 2 & 2 \\
\hline & Edges & 2 & 2 & $N A$ & 2 \\
\hline & Times (ms) & 0 & $N A$ & 0 & 0 \\
\hline \multirow{3}{*}{ Proc 12} & Classes & 186 & 186 & 188 & 186 \\
\hline & Edges & 262 & 262 & $N A$ & 262 \\
\hline & Times (ms) & 0 & $\overline{N A}$ & 16 & 0 \\
\hline \multirow{3}{*}{ Proc 123} & Classes & 958 & 958 & 1038 & 958 \\
\hline & Edges & 1506 & 1506 & $N A$ & 1506 \\
\hline & Times (ms) & 1 & $\overline{N A}$ & 391 & 3 \\
\hline \multirow{3}{*}{ Proc 1234} & Classes & 5.219 & 5.219 & 6.029 & 5.219 \\
\hline & Edges & 8.580 & 8.580 & $N A$ & 8.580 \\
\hline & Times (ms) & 31 & $N A$ & 2.719 & 38 \\
\hline \multirow{3}{*}{ Proc 12345} & Classes & 42.909 & 42.909 & 52.452 & 42.909 \\
\hline & Edges & 73.842 & 73.842 & $\overline{N A}$ & 73.842 \\
\hline & Times (ms) & 734 & $N A$ & 30000 & 786 \\
\hline
\end{tabular}

struction by using our algorithm is conforming with that of other tools. For this effect, we have considered the combination of the TPN shown in Figure 6. First, we started by testing the net Proc1, then by combining it with Proc2, and so on. The results of these experiments are reported in Table 2. The latter shows that when assuming the equality as equivalence relation, the computed graphs are identical whatever the tool we use. However, as the expression of a class is extended to the parameter $N E W$ in $O R I S$, the graphs computed by using this tool are coarser.

In the second series of tests, we aim at comparing the graph constructions defined in this paper with other fellow approaches. First of all, we have considered the ITPN given in Figure 3 while varying the intervals of transitions $t_{2}, t_{3}$ and $t_{6}$. The results of the tests are given in Table 3. We notice that for all the tests performed, our algorithms outperform the other tools in terms of computation time.

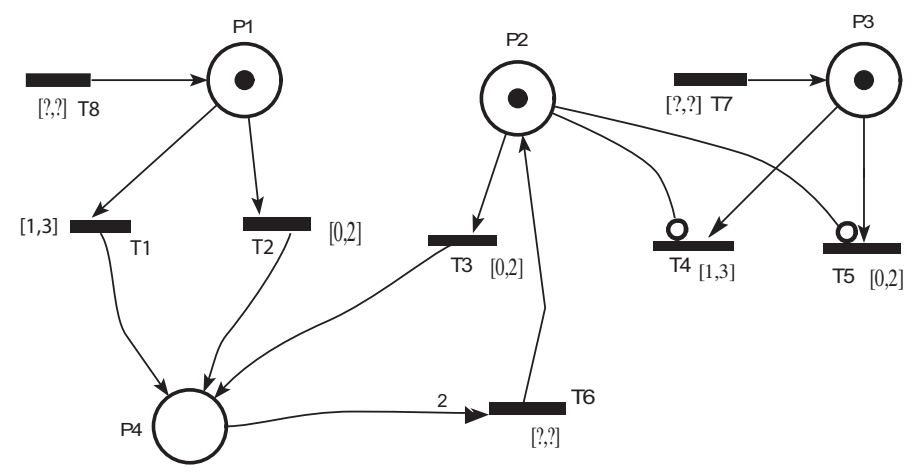

Figure 7: ITPN used in the experiments. 
Indeed, the optimization in the computation of $D B M$ systems makes it possible to speed up the construction of ours graph, faster than in the other tools. Comparing to ORIS (which is assumed to compute the tightest $D B M$ over-approximation as in $I T P N T$ ), the computation times are reduced approximatively by 30 . Concerning the tool $R O M E O$, besides that the computed graphs are less precise than ours, it seems also that the times needed for that are slower, even though they are not revealed. Moreover, the exact construction of the graph $G R$ needed high computation times to achieve (more than 1 minute in average), even failed in many cases as evidenced in the fourth test of Table.3. However, in some other cases (see the two final tests in Table.3), the exact construction succeeds to compute finite graphs whereas all $D B M$-over approximations techniques fail. This happens when the additional sequences (due to over-approximation), compute persistently new markings that stand unbounded in the graph.

Table 3: Results of experiments performed with ITPN of Figure 3

\begin{tabular}{|c|c|c|c|c|c|c|c|}
\hline \multirow[b]{2}{*}{ Examples } & \multirow{2}{*}{$\begin{array}{c}\text { TOOLS } \\
\text { Methods }\end{array}$} & \multirow{2}{*}{$\begin{array}{l}\text { TINA } \\
\text { K-grid }\end{array}$} & \multicolumn{2}{|c|}{ ROMEO } & \multicolumn{2}{|c|}{ ITPNT } & \multirow{2}{*}{$\begin{array}{l}\text { ORIS } \\
\text { DBM }\end{array}$} \\
\hline & & & Exact & $\mathrm{DBM}$ & $\widetilde{G R}(=)$ & $G R C(\simeq)$ & \\
\hline \multirow{3}{*}{$\begin{array}{c}\mathrm{t}_{2}[100,150] \\
\mathrm{t}_{3}[160,160] \\
\mathrm{t}_{6}[20,28]\end{array}$} & $\overline{\text { Classes }}$ & 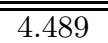 & $\bar{~} 4.489$ & 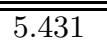 & $\bar{~} 5.378$ & $\bar{~} 5.098$ & 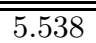 \\
\hline & Edges & 6.360 & 6.360 & 7.608 & 7.530 & 7.251 & $\overline{\mathrm{NA}}$ \\
\hline & Times $(\mathrm{ms})$ & 1632 & $\mathrm{NA}$ & $\mathrm{NA}$ & 51 & 45 & 1578 \\
\hline \multirow{3}{*}{$\begin{array}{c}\mathrm{t}_{2}[100,150] \\
\mathrm{t}_{3}[150,150] \\
\mathrm{t}_{6}[20,28]\end{array}$} & 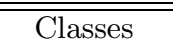 & $\overline{\overline{320}}$ & 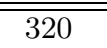 & 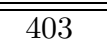 & 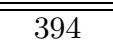 & 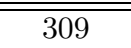 & $\overline{\overline{429}}$ \\
\hline & Edges & 460 & 460 & 575 & 562 & 446 & $\mathrm{NA}$ \\
\hline & Times $(\mathrm{ms})$ & 110 & $\mathrm{NA}$ & $\mathrm{NA}$ & 2 & 1 & 156 \\
\hline \multirow{3}{*}{$\begin{array}{c}\mathrm{t}_{2}[100,150] \\
\mathrm{t}_{3}[140,140] \\
\mathrm{t}_{6}[20,28] \\
\end{array}$} & $\overline{\text { Classes }}$ & $\overline{\overline{4.142}}$ & 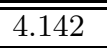 & 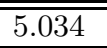 & 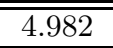 & $4 \overline{4.759}$ & 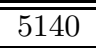 \\
\hline & Edges & 5.889 & 5.889 & 7.095 & 7.014 & 6.781 & $\overline{\mathrm{NA}}$ \\
\hline & Times $(\mathrm{ms})$ & 1502 & $\mathrm{NA}$ & $\mathrm{NA}$ & 41 & 37 & 1765 \\
\hline \multirow{3}{*}{$\begin{array}{c}\mathrm{t}_{3}[80,120] \\
\mathrm{t}_{5}[155,155] \\
\mathrm{t}_{6}[20,28]\end{array}$} & $\overline{\text { Classes }}$ & 28392 & $\overline{\mathrm{NF}}$ & $\overline{477.622}$ & 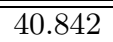 & $\overline{43.462}$ & $\overline{\mathrm{NF}}$ \\
\hline & edges & 41.452 & $\mathrm{NF}$ & 67.309 & 57.766 & 60.951 & $\mathrm{NF}$ \\
\hline & Times (ms) & 15703 & $\mathrm{NA}$ & $\mathrm{NA}$ & 878 & 886 & $\mathrm{NF}$ \\
\hline \multirow{3}{*}{$\begin{array}{c}\mathrm{t}_{2}[80,120] \\
\mathrm{t}_{3}[140,140] \\
\mathrm{t}_{6}[20,28]\end{array}$} & Classes & $\overline{7.018}$ & 7.018 & $\overline{~ 12.379}$ & $\overline{10.004}$ & $\overline{10.888}$ & $\overline{10.400}$ \\
\hline & Edges & 10.242 & 10.242 & 17.829 & 14.406 & 15.490 & $\overline{\mathrm{NA}}$ \\
\hline & Times $(\mathrm{ms})$ & 2834 & $\mathrm{NA}$ & $\mathrm{NA}$ & 178 & 162 & 3.516 \\
\hline \multirow{3}{*}{$\begin{array}{c}\mathrm{t}_{2}[100,150] \\
\mathrm{t}_{3}[135,135] \\
\mathrm{t}_{6}[20,28] \\
\end{array}$} & Classes & 111.351 & $\bar{~} 11.351$ & $\overline{16.354}$ & $\overline{15.178}$ & $\overline{16.646}$ & $\overline{15.318}$ \\
\hline & Edges & 15.649 & 15.649 & 22.230 & 20.486 & 23.225 & NA \\
\hline & Times $(\mathrm{ms})$ & 4907 & $\mathrm{NA}$ & $\mathrm{NA}$ & 220 & 236 & 4765 \\
\hline \multirow{3}{*}{$\begin{array}{c}\mathrm{t}_{2}[100,150] \\
\mathrm{t}_{3}[155,155] \\
\mathrm{t}_{6}[20,28]\end{array}$} & $\overline{\text { Classes }}$ & $\bar{~} 17.612$ & $\overline{17.612}$ & 21.857 & 21.626 & 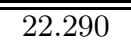 & 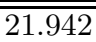 \\
\hline & Edges & 24.522 & 24.522 & 30.065 & 29.711 & 31.151 & $\overline{\mathrm{NA}}$ \\
\hline & Times $(\mathrm{ms})$ & 7951 & $\mathrm{NA}$ & $\mathrm{NA}$ & 285 & 289 & 5594 \\
\hline \multirow{3}{*}{$\begin{array}{c}\mathrm{t}_{2}[100,150] \\
\mathrm{t}_{3}[135,135] \\
\mathrm{t}_{6}[20,38]\end{array}$} & 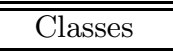 & $\bar{~} 12.874$ & $\overline{12.874}$ & $\overline{\mathrm{NF}}$ & $\overline{\mathrm{NF}}$ & $\overline{\mathrm{NF}}$ & $\overline{\mathrm{NF}}$ \\
\hline & Edges & 18.424 & 18.424 & $\mathrm{NF}$ & $\mathrm{NF}$ & $\mathrm{NF}$ & $\mathrm{NF}$ \\
\hline & Times $(\mathrm{ms})$ & 2340 & $\mathrm{NA}$ & $\mathrm{NF}$ & $\mathrm{NF}$ & $\mathrm{NF}$ & $\mathrm{NF}$ \\
\hline \multirow{3}{*}{$\begin{array}{c}\mathrm{t}_{2}[100,150] \\
\mathrm{t}_{3}[155,155] \\
\mathrm{t}_{6}[20,39]\end{array}$} & Classes & 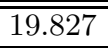 & 19.827 & $\overline{\mathrm{NF}}$ & $\overline{\mathrm{NF}}$ & $\overline{\mathrm{NF}}$ & $\overline{\mathrm{NF}}$ \\
\hline & Edges & 28.532 & 28.532 & $\mathrm{NF}$ & $\mathrm{NF}$ & $\mathrm{NF}$ & $\mathrm{NF}$ \\
\hline & Times $(\mathrm{ms})$ & 3635 & $\mathrm{NA}$ & $\mathrm{NF}$ & $\mathrm{NF}$ & $\mathrm{NF}$ & $\mathrm{NF}$ \\
\hline
\end{tabular}


Comparing to TINA which implements the K-grid based approximation, there are points in our favor and other against. As the results reported with TINA are obtained with the highest level grid, this construction achieves to build the exact graphs in almost all cases sensibly faster than $R O M E O$. However, although ours graphs are more coarse, they needed less time to be built (approximatively 25 times less). In other respects, the construction of the abstraction $\widetilde{G R C}$ seems to be more appropriate than that of $\widetilde{G R}$ when dealing with smaller graphs (less than 10.000 nodes). Otherwise the additional sequences due to the precision loss in $\widetilde{G R C}$ overwhelms the benefits of the contraction. However, the contraction is supposed to be more important in presence of conflicting transitions as we will see in the next experiments.

In the last experiments, we intend to advocate the benefits of building $\widetilde{G R C}$ rather than $\widetilde{G R}$ when dealing with both conflicting and inhibiting transitions. For this effect, we have considered the ITPN given in Figure 7 while varying the intervals of transitions $t_{6}, t_{7}$ and $t_{8}$; the results of these experiments are reported in Table 4 .

All the experiments show that the graph computation times are in favor of our constructions, yet more when computing the abstraction $\widehat{G R C}$. Furthermore, in the first two experiments, the construction of the graph $\widetilde{G R}$ succeeds to build the exact graph, unlike the $D B M$ over-approximations implemented in $R O M E O$.

On the other side, the construction of the abstraction $\widetilde{G R C}$ achieves to reduce significantly the size of the graphs as well as their computation effort. Moreover, this contraction has provided very compact graphs which are even smaller than those computed by the exact approach. However, this does not mean that the graphs $\widetilde{G R C}$ are more precise in the approximation than $\widetilde{G R}$, but denote that many classes that stand unequal in $\widetilde{G R}$ and $G R$ are bisimilar indeed. The application of the equivalency $\simeq$ makes it possible to gather these bisimilar classes, and therefore to compact sensibly the graphs.

Furthermore, we notice that for the last four tests in Table.4, the exact computation of the graph as well as the K-grid approximation fail to build the graphs, unlike $D B M$ over-approximation approaches. This happens when the number of different polyhedra computed in the exact graph is unbounded, while the number of $D B M$ systems obtained by approximation is always bounded.

\section{Conclusion}

We have proposed in this paper an efficient algorithm to construct the tighter $D B M$ over-approximation of the state class graph of preemptive systems modeled by using the ITPN model. Similarly as in [7][15], our approach is based on overapproximating the polyhedron of each reachable class by relaxing its non $D B M$ constraints. For this effect, we have proposed to shun the computation of the intermediary polyhedra, and have provided an algorithm that computes efficiently and straightforwardly the full $D B M$ system in its normal form. We have thereby 
Table 4: Results of experiments performed with ITPN of Figure 7.

\begin{tabular}{|c|c|c|c|c|c|c|}
\hline \multirow[b]{2}{*}{ Examples } & \multirow{2}{*}{$\begin{array}{c}\text { TOOLS } \\
\text { Methods }\end{array}$} & \multirow{2}{*}{$\begin{array}{l}\text { TINA } \\
\text { K-grid } \\
\end{array}$} & \multicolumn{2}{|c|}{ ROMEO } & \multicolumn{2}{|c|}{ ITPNT } \\
\hline & & & Exact & DBM & $\widetilde{G R}(=)$ & $G R C(\simeq)$ \\
\hline \multirow{3}{*}{$\begin{array}{c}\mathrm{t}_{6}[2,5] \\
\mathrm{t}_{7}[22,35] \\
\mathrm{t}_{8}[20,30]\end{array}$} & Classes & 1.035 & 1.035 & 1.318 & 1.035 & 842 \\
\hline & Edges & 1.830 & 1.830 & 2.323 & 1.830 & 1.471 \\
\hline & Times(ms) & 312 & $\mathrm{NA}$ & $\mathrm{NA}$ & 8 & 4 \\
\hline \multirow{3}{*}{$\begin{array}{c}\mathrm{t}_{6}[2,5] \\
\mathrm{t}_{7}[12,15] \\
\mathrm{t}_{8}[10,20] \\
\end{array}$} & Classes & 750 & 750 & 1.685 & 750 & 742 \\
\hline & Edges & 1.363 & 1.363 & 3.106 & 1.363 & 1.356 \\
\hline & Times(ms) & 219 & $\mathrm{NA}$ & $\mathrm{NA}$ & 4 & 3 \\
\hline \multirow{3}{*}{$\begin{array}{c}\mathrm{t}_{6}[4,8] \\
\mathrm{t}_{7}[14,20] \\
\mathrm{t}_{8}[10,20]\end{array}$} & Classes & 2.346 & 2.346 & 3.398 & 2.402 & 1.880 \\
\hline & Edges & 4.969 & 4.969 & 7.198 & 5.090 & 4.118 \\
\hline & Times $(\mathrm{ms})$ & 1312 & $\mathrm{NA}$ & $\mathrm{NA}$ & 19 & 12 \\
\hline \multirow{3}{*}{$\begin{array}{c}\mathrm{t}_{6}[4,10] \\
\mathrm{t}_{7}[16,22] \\
\mathrm{t}_{8}[10,18] \\
\end{array}$} & Classes & 3.203 & 3.203 & 4.451 & 3.238 & 2.648 \\
\hline & Edges & 6.603 & 6.603 & 9.184 & 6.756 & 5.758 \\
\hline & Times(ms) & 1.594 & $\mathrm{NA}$ & $\mathrm{NA}$ & 28 & 19 \\
\hline \multirow{3}{*}{$\begin{array}{c}\mathrm{t}_{6}[4,8] \\
\mathrm{t}_{7}[16,22] \\
\mathrm{t}_{8}[10,15] \\
\end{array}$} & Classes & $\mathrm{NF}$ & $\mathrm{NF}$ & 20.638 & 19.739 & 16.981 \\
\hline & Edges & $\mathrm{NF}$ & $\mathrm{NF}$ & 46.216 & 43.378 & 38.338 \\
\hline & Times $(\mathrm{ms})$ & $\mathrm{NA}$ & $\mathrm{NF}$ & $\mathrm{NA}$ & 426 & 363 \\
\hline \multirow{3}{*}{$\begin{array}{c}\mathrm{t}_{6}[4,10] \\
\mathrm{t}_{7}[16,25] \\
\mathrm{t}_{8}[10,18] \\
\end{array}$} & Classes & $\mathrm{NF}$ & $\mathrm{NF}$ & 20.875 & 20.048 & 17.272 \\
\hline & Edges & $\mathrm{NF}$ & $\mathrm{NF}$ & 46.945 & 44.261 & 39.137 \\
\hline & Times $(\mathrm{ms})$ & $\mathrm{NA}$ & $\mathrm{NF}$ & $\mathrm{NA}$ & 440 & 361 \\
\hline \multirow{3}{*}{$\begin{array}{c}\mathrm{t}_{6}[4,10] \\
\mathrm{t}_{7}[16,25] \\
\mathrm{t}_{8}[10,20] \\
\end{array}$} & Classes & $\mathrm{NF}$ & $\mathrm{NF}$ & 20.451 & 19.636 & 16.891 \\
\hline & Edges & $\mathrm{NF}$ & $\mathrm{NF}$ & 45.993 & 43.338 & 38.237 \\
\hline & Times $(\mathrm{ms})$ & $\mathrm{NA}$ & $\mathrm{NF}$ & $\mathrm{NA}$ & 425 & 342 \\
\hline \multirow{3}{*}{$\begin{array}{c}\mathrm{t}_{6}[4,10] \\
\mathrm{t}_{7}[16,27] \\
\mathrm{t}_{8}[10,28]\end{array}$} & Classes & $\mathrm{NF}$ & $\mathrm{NF}$ & 19.092 & 18.481 & 15.915 \\
\hline & Edges & $\mathrm{NF}$ & $\mathrm{NF}$ & 43.116 & 40.941 & 36.085 \\
\hline & Times $(\mathrm{ms})$ & $\mathrm{NA}$ & $\mathrm{NF}$ & $\mathrm{NA}$ & 403 & 324 \\
\hline
\end{tabular}


succeeded to remove the drawbacks due to the manipulation of the intermediary polyhedra, and improved significantly the graph construction by removing the cost of the normalization and the minimization of the $D B M$ system.

Then, in the second part of this work, we have proposed a new approach to compute an abstraction of the state space of an ITPN. For this effect, we showed that by relaxing a little bit in the precision of the $D B M$ over-approximation, we can compute graphs that can be more appropriate, in certain cases, to model-check the linear properties of the ITPN. We have discussed how this construction can be improved yet more by leaving out all the distances that are useless for the class computation process. Hence, we have put forward an equivalence relation that makes it possible to contract sensibly the size of the graphs as well as to reduce the effort of their computation. Experimental results have been reported to advocate the benefits of both constructions.

\section{References}

[1] Avis, D., K. Fukuda and S. Picozzi, On canonical representations of convex polyhedra. First International Congress of Mathematical Software (2002), pp. $350-360$.

[2] R. Alur, C. Courcoubetis, N. Halbwachs, T. A. Henzinger, P-H. Ho, X. Nicollin, A. Olivero, J. Sifakis, S. Yovine: The Algorithmic Analysis of Hybrid Systems. Theor. Comput. Sci. 138(1): 3-34 (1995)

[3] Bernard Berthomieu, Miguel Menasche: An Enumerative Approach for Analyzing Time Petri Nets. IFIP Congress 1983: 41-46

[4] B. Berthomieu, and M. Diaz. "Modeling and verification of time dependant systems using Time Petri Nets". IEEE TSE, 17(3):(259-273), March 1991.

[5] Bernard Berthomieu, Didier Lime, Olivier H. Roux, François Vernadat: Reachability Problems and Abstract State Spaces for Time Petri Nets with Stopwatches. Discrete Event Dynamic Systems 17(2): 133-158 (2007).

[6] Hanifa Boucheneb, Hind Rakkay: A More Efficient Time Petri Net State Space Abstraction Useful to Model Checking Timed Linear Properties. Fundam. Inform. 88(4): 469-495 (2008).

[7] G. Bucci, A. Fedeli, L. Sassoli, and E.Vicario. Timed State Space Analysis of Real-Time Preemptive Systems. IEEE TSE, Vol 30, No. 2, Feb 2004.

[8] Dill, D.L.: Timing assumptions and verification of finite-state concurrent systems; Workshop Automatic Verification Methods for Finite-State Systems. Vol 407. (1989) 197-212.

[9] P. Merlin. "A study of the recoverability of computer system". PhD thesis Dep. Comp. Science, Uni. California, Irvine, 1974. 
[10] F. Cassez and K.G. Larsen. The Impressive Power of Stopwatches. LNCS, vol. 1877, pp. 138-152, Aug. 2000.

[11] Thomas A. Henzinger: The Theory of Hybrid Automata. LICS 1996: 278-292

[12] Thomas A. Henzinger, Rupak Majumdar, Jean-François Raskin: A classification of symbolic transition systems. ACM Trans. Comput. Log. 6(1): 1-32 (2005)

[13] D.Lime, and O.H.Roux. Expressiveness and analysis of scheduling extended time Petri nets. In 5th IFAC International Conference on Fieldbus Systems and their Applications, (FET'03), Elsevier Science, July, 2003.

[14] Morgan Magnin, Didier Lime, Olivier H. Roux: An Efficient Method for Computing Exact State Space of Petri Nets With Stopwatches. Electr. Notes Theor. Comput. Sci. 144(3): 59-77 (2006).

[15] Olivier H. Roux, Didier Lime: Time Petri Nets with Inhibitor Hyperarcs. Formal Semantics and State Space Computation. ICATPN 2004: 371-390.

[16] ORIS TOOL:http://www.stlab.dsi.unifi.it/oris/index.html.

[17] ROMEO TOOL http://romeo.rts-software.org.

[18] TINA Tool http://www.laas.fr/tina/.

Received 13th January 2010 